TITLE:

\title{
An illustrated and annotated checklist of fishes on Kitami- Yamato Bank, southern Sea of Okhotsk
}

\section{AUTHOR(S):}

TOHKAIRIN, Akira; HAMATSU, Tomonori; YOSHIKAWA, Akane; KAI, Yoshiaki; NAKABO, Tetsuji

\section{CITATION:}

TOHKAIRIN, Akira ... [et al]. An illustrated and annotated checklist of fishes on KitamiYamato Bank, southern Sea of Okhotsk. Publications of the Seto Marine Biological Laboratory 2015, 43: 1-29

\section{ISSUE DATE:}

2015-09-15

URL:

http://hdl.handle.net/2433/193238

RIGHT: 
Publ. Seto Mar. Biol. Lab., 43: 1-29, 2015

\title{
An illustrated and annotated checklist of fishes on Kitami-Yamato Bank, southern Sea of Okhotsk
}

\author{
AKIRA TOHKAIRIN ${ }^{1}$, TOMONORI HAMATSU ${ }^{2}$, AKANE YOSHIKAWA $^{3}$, YOSHIAKI KAI $^{4}$ and \\ TETSUJI NAKABO 5 \\ ${ }^{1}$ Graduate School of Agriculture, Division of Applied Biosciences, Kyoto University \\ Kitashirakawa oiwake-cho, Sakyo, Kyoto 606-8502, Japan \\ E-mail: tokairin.akira.84n@st.kyoto-u.ac.jp \\ ${ }^{2}$ Hokkaido National Fisheries Research Institute, Fisheries Research Agency \\ Katsurakoi, Kushiro, Hokkaido 085-0802, Japan \\ ${ }^{3}$ Department of Bioresource Science, Faculty of Agriculture, Kyoto University \\ Kitashirakawa oiwake-cho, Sakyo, Kyoto 606-8502, Japan \\ ${ }^{4}$ Maizuru Fisheries Research Station, Field Science Education and Research Center, Kyoto University \\ Nagahama, Maizuru, Kyoto 625-0086, Japan \\ ${ }^{5}$ The Kyoto University Museum, Kyoto University \\ Yoshida, Sakyo, Kyoto 606-8501, Japan
}

\begin{abstract}
An annotated checklist of benthic fishes on the western part of Kitami-Yamato Bank off northeastern Hokkaido, Japan in the southern Sea of Okhotsk, is presented based on voucher specimens with accompanying color photographs. All material was collected in late April, 2013 and 2014 by the bottom trawl net, a total of 450 specimens being classified into 92 species representing 21 families. These include Bathyraja trachouros, the first record from the Sea of Okhotsk, and Careproctus segaliensis, only the second confirmed record of the species.
\end{abstract}

Key words: Sea of Okhotsk, Kitami-Yamato Bank, ichthyofauna, benthic fish, bottom trawl net, voucher specimen

\section{Introduction}

The Sea of Okhotsk, a marginal, semi-enclosed sea in the western North Pacific Ocean, is bordered by the Okhotsk Coast (Siberia), Kamchatka Peninsula, Kuril Islands and Sakhalin Island, Russia, and Hokkaido, Japan (Text-fig. 1). Although the mean depth is $777 \mathrm{~m}$, but the northern and western parts are shallow, gradually sloping toward the Kuril Basin and reaching a maximum depth of 3,657 $\mathrm{m}$ in the southeastern region (Nishimura 1983; Tyler 2002). The Kuril Islands from a partial barrier between the Sea of Okhotsk and the Pacific Ocean, and the depths between adjacent islands being relatively shallow with most not exceeding $100 \mathrm{~m}$, except for the Krusenstern Strait (1,920 m depth) and the Bussol Strait (2,318 m depth) (Nishimura 1983). Connected by very shallow narrow straits [the Mamiya Strait (15 m depth) and Soya Strait (53 m depth)] with the Sea of Okhotsk (Tyler 2002), the Sea of Japan is also a semi-enclosed sea on the margin of 
the western North Pacific Ocean. Such marginal seas have been considered as having been isolated due to lowered sea levels during glacial periods by several phylogeographic studies (e.g., Liu et al. 2007; Xu and Chu 2012). On the other hand, the Sea of Okhotsk is believed to have never been completely isolated from the Pacific Ocean during glacial periods, because of the great depths of the adjoining straits (Bezverkhniy et al. 2002). In any case, the complex geological history and structure of the marginal seas in the western North Pacific Ocean (reviewed in Wang 1999) are considered to have been important in generating biodiversity (Briggs and Bowen 2012).

Although the detailed geological history of the Sea of Okhotsk is still unknown, it has been suggested that the sea was covered by seasonal sea-ice during the last glacial period (Gorbarenko et al. 2002; Harada et al. 2006), with productivity in the southeast region being extremely high (Gorbarenko et al. 2002). Furthermore, the deep straits in the Kuril Island chain allow water and species exchange with the Pacific Ocean. Accordingly, the Sea of Okhotsk shares many species with the Pacific Ocean, including cold-adapted benthic species (Nishimura 1983; Tyler 2002). In contrast, Nishimura (1974) considered seven deep-sea species/subspecies endemic to the Sea of Japan to share common ancestry with species/subspecies distributed in the Sea of Okhotsk and/or the Bering Sea, suggesting a close relationship between the Seas of Okhotsk and Japan.

In this context, the ichthyofauna of the Sea of Okhotsk has been variously investigated over the years. In the first half of the 20th century, several reports of ichthyofauna of the Sea of Okhotsk and adjacent waters were published by Russian ichthyologists (e.g., Soldatov and Lindberg 1930; Taranetz 1937; Schmidt 1950), followed by major publications on the fishes of the Sea of Japan and adjacent waters, including the southern Sea of Okhotsk, were published (Lindberg and Legeza 1959, 1965; Lindberg and Krasyukova 1969, 1975, 1987; Lindberg and Fedorov 1993; Lindberg et al. 1997). These reports were based partly on the voucher specimens deposited in the Zoological Institute of the Russian Academy of Sciences, St. Petersburg, Russia (ZIN), but were mostly based on literature records. In addition, many checklists including fishes of the Sea of Okhotsk have been published [e.g., Parin (2001, 2003), Parin et al. (2002), and Fedorov et al. (2003)], but included no specimen or locality details. In addition, Ueno (1971) and Maeda and Tsutsui (2003) summarized the ichthyofauna of the Hokkaido, including the Sea of Okhotsk coast, but included neither voucher specimens nor literature citations.

On the other hand, Amaoka et al. (1983) reported 80 species of deep-sea fishes from the southern Sea of Okhotsk on the basis of the voucher specimens and Shinohara et al. (2012) published a preliminary list of fishes (138 species) from Nemuro Strait (between the Sea of Okhotsk and the Pacific Ocean), also based on voucher specimens. Nevertheless, the number of voucher-based list from the Sea of Okhotsk are still limited. This list (preliminary only) includes voucher specimens and photographs of benthic fishes collected by the bottom trawl net on the Kitami-Yamato Bank, the southern Sea of Okhotsk. The latter study area is broader than that treated by Amaoka et al. (1983), covering the western part of the Bank, a major fishing ground off Hokkaido on which many fisheries surveys have been conducted (Amaoka et al. 1983).

\section{Materials and Methods}

Fishes were collected during the trawl surveys conducted by the Hokkaido National Fisheries Research Institute, Fisheries Research Agency, Kushiro, Japan, on the R/V Kaiyo-maru No. 5 (495 gross-tonnage), Ocean Engineering Co., Ltd. , Tokyo, Japan, (chartered by Hokkaido National Fisheries Research Institute, Fisheries Research Agency) in late April, 2013 and 2014. The collection stations and survey data are shown in Text-fig. 1 and Table 1. At the each station, the bottom trawl net (cod-end mesh size of $15 \mathrm{~mm}$ ) was towed at a ground speed of three knots for 30 minutes. 
All specimens are deposited in the fish collection of Kyoto University, Kyoto, Japan (FAKU). The systematic arrangement of families follows Nakabo (2013). Specimens in each family are arranged in alphabetical order by species. Author(s), and scientific and Japanese names generally follow Nakabo (2013), with some modifications following recent published studies (Coulson et al. 2006; Ishihara et al. 2012; Higuchi et al. 2014; Nazarkin et al. 2014). Each voucher specimen includes registration number, standard length (abbreviated as SL) or total length (abbreviated as TL), trawl survey station (Text-fig. 1, Table 1), and the collection date. Tissues for future DNA analysis were taken and preserved at FAKU, except for the specimens indicated by an asterisk (*).

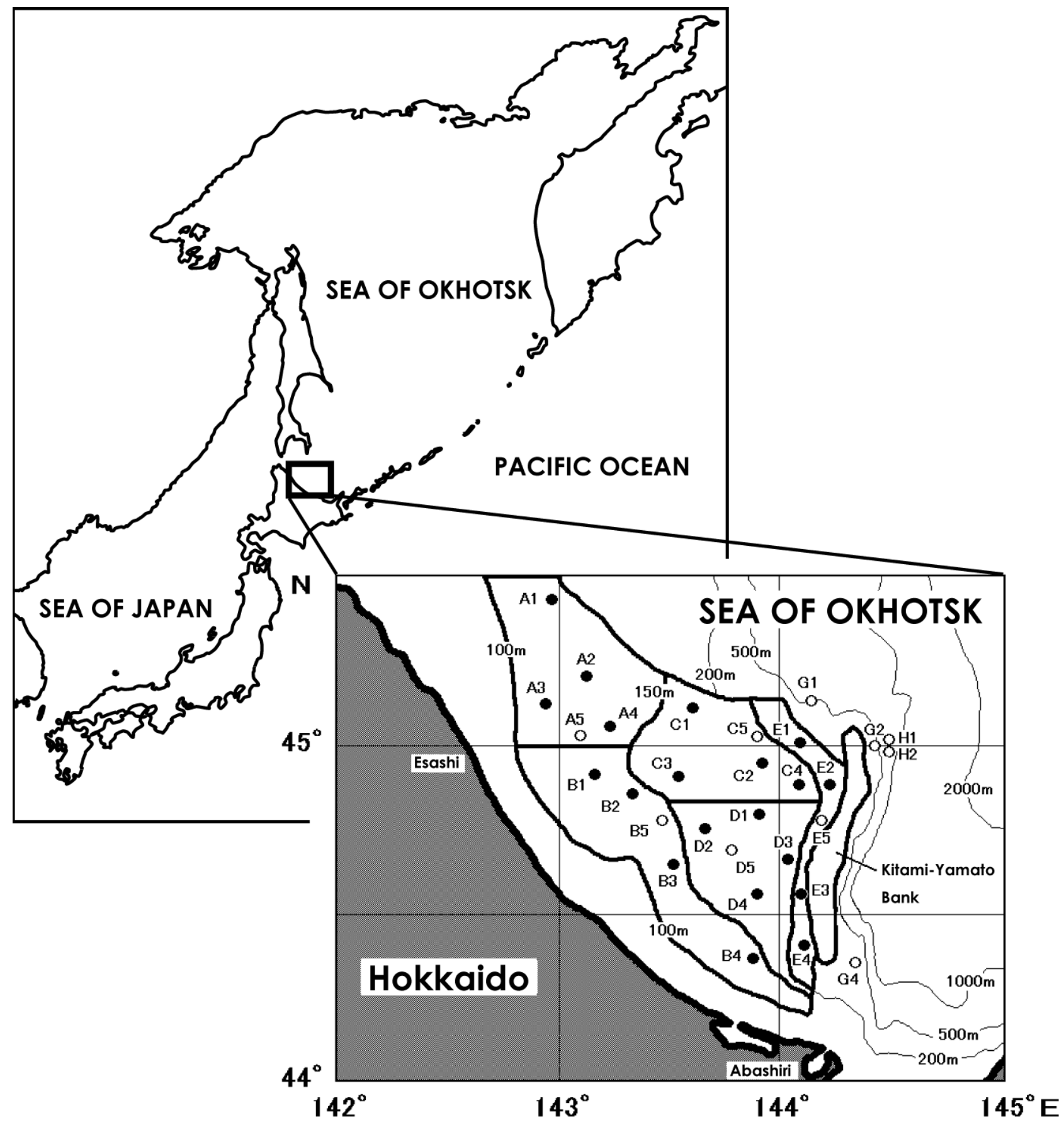

Text-fig. 1. Location of investigation area. Circles indicate stations surveyed by R/V Kaiyo-maru No. 5 in 2013-2014. White circles indicate reserve sites. 


\section{Results}

\section{Order Rajiformes \\ Family Rajidae}

1. Bathyraja matsubarai (Ishiyama, 1952) [Japanese name: Matsubara-ei] (Plate I, Figs. 1, 2)

FAKU 201463, only tissue preserved, G1, 21 Apr. 2014.

2. Bathyraja smirnovi (Soldatov and Palvenko, 1915) [Japanese name; Dobu-kasube] (Plate I, Fig. 3)

FAKU 200793, 356.0 mm TL, G2, 20 Apr. 2013; FAKU 201462, only tissue preserved, C5, 20 Apr. 2014; FAKU 201464, only tissue preserved, G1, 21 Apr. 2014.

\section{Bathyraja trachouros (Ishiyama, 1958) [Japanese name: Zara-kasube] (Plate I, Fig. 4)}

FAKU 200676, 279.9 mm TL, G4, 23 Apr. 2013.

Remarks: The single specimen was characterized by the following characters, which agreed well with the original description of Ishiyama (1958): dermal denticles distributed over dorsal surface of disk; ventral surface was entirely smooth; nuchal thorns present, but barely discernable; shoulder spine was distinct on both sides; enlarged thorns were arranged in a longitudinal row along mesial line on tail; dermal folds on both sides of posterior tail. The species has been recorded from the Pacific coast of northern Japan and Taiwan (Ebert et al. 2013; Hatooka et al. 2013). This is the first record of the species from the Sea of Okhotsk.

\section{Bathyraja violacea (Suvorov, 1935) [Japanese name: Kitano-kasube] ( Plate I, Fig. 5)}

No voucher specimens, G4, 23 Apr. 2013.

\section{Beringraja pulchra (Liu, 1932) [Japanese name: Megane-kasube] (Plate I, Fig. 6)}

FAKU 201459-201461, only tissue preserved, A3, 17 Apr. 2014; FAKU 201465, only tissue preserved, D2, 22 Apr. 2014; FAKU 201466-201471, only tissue preserved, A3(2), 23 Apr. 2014; FAKU 201472, only tissue preserved, A5(2), 23 Apr. 2014; FAKU 201473, only tissue preserved, D4, 22 Apr. 2014; FAKU 201474, 201475, only tissue preserved, D5, 22 Apr. 2014; FAKU 201476, only tissue preserved, B1(2), 23 Apr. 2014.

Remarks: Recently, Ishihara et al. (2012) described a new genus Beringraja, containing Beringraja binoculata (Girard, 1935) and B. pulchra, on the basis of egg capsule morphology. Although Hatooka et al. (2013) contained to refer the latter species to the genus Raja, the taxonomy adopted here follows Ishihara et al. (2012).

\section{Order Clupeiformes \\ Family Clupeidae}

6. Clupea pallasii Valenciennes, 1847 [Japanese name: Nishin] (Plate II, Fig. 1)

FAKU 200419, 200420, 73.4-150.7 mm SL, C2, 20 Apr. 2103; FAKU 200548, 147.5 mm SL, E5, 21 Apr. 2013; FAKU 200599-200601, 139.3-168.8 mm SL, A1, 17 Apr. 2013.

\section{Order Argentiniformes \\ Family Microstomatidae}

7. Leuroglossus schmidti Rass, 1955 [Japanese name: Togari-ichimonji-iwashi] (Plate II, Fig. 2) FAKU 200388, 200389, 115.0-134.0 mm SL, G1, 19 Apr. 2013. 


\section{FISH OF KITAMI-YAMATO BANK, SOUTHERN SEA OF OKHOTSK}

8. Lipolagus ochotensis (Schmidt, 1938) [Japanese name: Soko-iwashi] (Plate II, Fig. 3) FAKU 200387, 139.6 mm SL, G1, 19 Apr. 2013.

\section{Order Salmoniformes \\ Family Osmeridae}

9. Mallotus villosus (Müllar, 1776) [Japanese name: Karafuto-shishamo] (Plate II, Fig. 4)

FAKU 200576, 154.0 mm SL, A4, 18 Apr. 2013; FAKU 200594-200598, 138.7-152.4 mm SL, A1, 17 Apr. 2013.

10. Osmerus dentex Steindachner and Kner, 1870 [Japanese name: Kyūriuo] (Plate II, Fig. 5)

FAKU 200421, 189.6 mm SL, C2, 20 Apr. 2013; FAKU 20577-200579, 132.8-201.8 mm SL, A4, 18 Apr. 2013; FAKU 200681, 122.5 mmSL, A5, 17 Apr. 2013.

\section{Order Myctophiformes \\ Family Myctophidae}

11. Nannobrachium regale (Gilbert, 1891) [Japanese name: Mikado-hadaka] (Plate II, Fig. 6)

FAKU 200557, 183.2 mm SL, G4, 23 Apr. 2013.

12. Stenobrachius nannochir (Gilbert, 1890) [Japanese name: Sekki-hadaka] (Plate II, Fig. 7)

FAKU 200385, 200386, 96.1-97.7 mm SL, G1, 19 Apr. 2013.

\section{Order Gadiformes \\ Family Moridae}

13. Laemonema longipes Schimidt, 1938 [Japanese name: Itohikidara] (Plate II, Fig. 8)

FAKU 200790-200792, 141.2-213.0 mm SL, G2, 20 Apr. 2013.

\section{Family Gadidae}

14. Eleginus gracilis (Tilesius, 1810) [Japanese name: Komai] (Plate II, Fig. 9)

FAKU 200538, 200539, 122.3-132.0 mm SL, D1, 21 Apr. 2013; FAKU 200590, 200606, 200607, 105.4206.4 mm SL, A1, 17 Apr. 2013; FAKU 200608-200611, 132.6-142.1 mm SL, C3, 18 Apr. 2013.

15. Gadus chalcogrammus Pallas, 1814 [Japanese name: Suketō-dara] (Plate II, Fig. 10)

FAKU 200562, 99.0 mm SL, E1, 19 Apr. 2013.

Remarks: This species had been recognized as a member of the genus Theragra (e.g., Schmidt 1950; Nakabo and Kai 2013c). However, Coulson et al. (2006), subsequently followed by Page et al. (2013), recently transferred it to the genus Gadus on the basis of a molecular analysis.

16. Gadus macrocephalus Tilesius, 1810 [Japanese name: Ma-dara] (Plate II, Fig. 11)

FAKU 200549, 262.5 mm SL, E5, 21 Apr. 2013.

Order Gasterosteiformes

Family Gasterosteidae 
17. Gasterosteus nipponicus Higuchi et al., 2014 [Japanese name: Nihon-itoyo] (Plate III, Fig. 1)

FAKU 200688, 200689, 62.9-66.8 mm SL, A2, 17 Apr. 2013; FAKU 201424-201430, 56.9-66.4 mm SL, A2(2), 23 Apr. 2014.

Remarks: This species was formerly considered as one of the two forms of Gasterosteus aculeatus Linnaeus, 1758, i.e., "Sea of Japan form of G. aculeatus" sensu Hosoya (2000). However, molecular and morphological evidence for their status as separate species was presented by Higuchi and Goto (1996) and Yamada et al. (2001), with the "Sea of Japan form of G. aculeatus" being described as a new species, Gasterosteus nipponicus, by Higuchi et al. (2014). The present specimens were identified as G. nipponicus by the following characters: lateral plates complete, abruptly reducing in size above the anus (depth of lateral plate above the anus $<60 \%$ that of the deepest plate); caudal keels thin, membranous (Higuchi et al. 2014).

\section{Order Perciformes \\ Family Sebastidae}

18. Sebastes glaucus Hilgendorf, 1880 [Japanese name: Kuro-menuke] (Plate III, Fig. 2)

FAKU 200731, 191.4 mm SL, D5, 22 Apr. 2013; FAKU 200794, 265.9 mm SL, D3, 21 Apr. 2013.

\section{Family Sebastolobidae}

19. Sebastolobus macrochir (Günther, 1877) [Japanese name: Kichiji] (Plate III, Fig. 3)

FAKU 200753, 162.3 mm SL, G2, 20 Apr. 2013.

\section{Family Hexagrammidae}

20. Hexagrammos lagocephalus (Pallas, 1810) [Japanese name: Usagi-ainame] (Plate III, Fig. 4)

No voucher specimens, G1, 19 Apr. 2013.

21. Pleurogrammus azonus Jordan and Metz, 1913 [Japanese name: Hokke] (Plate III, Fig. 5)

FAKU 200797, 210.2 mm SL, D2, 21 Apr. 2013.

\section{Family Trichodontidae}

22. Arctoscopus japonicus (Steindachner, 1881) [Japanese name: Hatahata] (Plate III, Fig. 6)

FAKU 201377, 178.9 mm SL, B2, 18 Apr. 2014; FAKU 201421, 89.9 mm SL, D2, 22 Apr. 2014.

\section{Family Hemitripteridae}

23. Blepsias bilobus Cuvier in Cuvier and Valenciennes, 1829 [Japanese name: Hokake-anahaze] (Plate III, Fig. 7)

FAKU 201355, 134.7 mm SL, B1, 18 Apr. 2014; FAKU 201394, 201395, 154.6-171.4 mm SL, B3, 19 Apr. 2014.

24. Hemitripterus villosus (Pallas, 1814) [Japanese name: Kemushi-kajika] (Plate III, Fig. 8)

FAKU 201362, 165.8 mm SL, A3, 17 Apr. 2014; FAKU 201423, 168.8 mm SL, A2(2), 23 Apr. 2014; FAKU 201440, 189.3 mm SL, C1, 20 Apr. 2014.

\section{Family Cottidae}




\section{FISH OF KITAMI-YAMATO BANK, SOUTHERN SEA OF OKHOTSK}

25. Enophrys diceraus (Pallas, 1787) [Japanese name: Oni-kajika] (Plate IV, Fig. 1)

FAKU 200796, 189.0 mm SL, D2, 21 Apr. 2013.

26. Gymnocanthus detrisus Gilbert and Burke, 1912 [Japanese name: Sebiro-kajika] (Plate IV, Fig. 2)

FAKU 200401, 85.9 mm SL, A1, 17 Apr. 2013; FAKU 200575, 79.8 mm SL, A4, 18 Apr. 2013; FAKU 200663, 104.4 mm SL, A2, 17 Apr. 2013.

27. Gymnocanthus herzensteini Jordan and Starks, 1904 [Japanese name: Tsumaguro-kajika] (Plate IV, Fig. 3)

FAKU 201358, 194.5 mm SL, A5, 18 Apr. 2014; FAKU 201396, 204.0 mm SL, D4, 22 Apr. 2014.

28. Hemilepidotus gilberti Jordan and Starks, 1904 [Japanese name: Yokosujikajika] (Plate IV, Fig. 4)

FAKU 200712-200714, 76.6-116.3 mm SL, A3, 17 Apr. 2013; FAKU 200800, 89.1 mm SL, D4, 23 Apr. 2013.

29. Hemilepidotus papilio (Bean, 1880) [Japanese name: Kujaku-kajika] (Plate IV, Figs. 5, 6)

FAKU 200455, 218.6 mm SL, female, C4, 20 Apr. 2013; FAKU 200754, 246.9 mm SL, male, E2, 20 Apr. 2013.

30. Icelus cataphractus (Pavlenko, 1910) [Japanese name: Kōrikajika] (Plate IV, Fig. 7)

FAKU 200570-200572, 90.2-121.9 mm SL, E1, 19 Apr. 2013; FAKU 200662, 177.7 mm SL, A2, 17 Apr. 2013; FAKU 200682, 159.4 mm SL, A5, 17 Apr. 2013; FAKU 200741, 131.7 mm SL, B1, 19 Apr. 2013; FAKU 200757-200759, 108.8-120.3 mm SL, B4, 24 Apr. 2013.

31. Icelus gilberti Taranetz, 1936 [Japanese name: Daruma-kōrikajika] (Plate IV, Fig. 8)

FAKU 201380, 83.7 mm SL, B4, 19 Apr. 2014.

32. Icelus ochotensis Schmidt, 1927 [Japanese name: Kobu-kōrikajika] (Plate IV, Fig. 9)

FAKU 201397, 82.3 mm SL, C5, 20 Apr. 2014.

33. Myoxocephalus jaok (Cuvier in Cuvier and Valenciennes, 1829) [Japanese name: Oku-kajika] (Plate IV, Fig. 10)

FAKU 200661, 245.0 mm SL, A2, 17 Apr. 2013; FAKU 200756, 278.7 mm SL, B4, 24 Apr. 2013.

34. Myoxocephalus polyacanthocephalus (Pallas, 1814) [Japanese name: Toge-kajika] (Plate IV, Fig. 11) FAKU 200755, 233.6 mm SL, E2, 20 Apr. 2013

35. Taurocottus bergii Soldatov and Pavlenko, 1915 [Japanese name: Kiri-kajika] (Plate IV, Fig. 12)

FAKU 200732, 122.6 mm SL, D5, 22 Apr. 2013; FAKU201422, 107.6 mm SL, D5, 22 Apr. 2014.

36. Triglops pingelii Reinhardt, 1837 [Japanese name: Hokkyoku-kajika] (Plate V, Fig. 1)

FAKU 200573, 200574, 150.3-153.0 mm SL, A4, 18 Apr. 2013; FAKU 200683, 152.2 mm SL, A5, 17 Apr. 2013; FAKU 201383, 148.8 mm SL, A2, 16 Apr. 2014.

37. Triglops scepticus Gilbert, 1896 [Japanese name: Nirami-kajika] (Plate V, Fig. 2)

FAKU 200702-200706, 137.2-152.0 mm SL, B3, 22 Apr. 2013; FAKU 200738-200740, 129.0-147.8 mm SL, B1, 18 Apr. 2013. 


\section{TOHKAIRIN, A. ET AL.}

\section{Family Psychrolutidae}

38. Dasycottus setiger Bean, 1890 [Japanese name: Ganko] (Plate V, Fig. 3)

FAKU 200612, 89.8 mm SL, C3, 18 Apr. 2013; FAKU 200664, 99.7 mm SL, A2, 17 Apr. 2013.

39. Malacocottus zonurus Bean, 1890 [Japanese name: Kobushi-kajika] (Plate V, Figs. 4, 5)

FAKU 200378-200384, 200470, 33.8-144.0 mm SL, G1, 19 Apr. 2013; FAKU 201382, 73.2 mm SL, B4, 19 Apr. 2014; FAKU 201398-201400, 39.0-62.9 mm SL, G1, 21 Apr. 2014; FAKU 201435-201439, 39.165.9 mm SL, E1, 21 Apr. 2014.

Remarks: The specimens were identified following the keys provided by Nakabo and Kai (2013d), including characters such as "prickle-like scales on head" and "a prominent spine present on base of second preopercular spin". However, the small specimens (< 50 mm SL: FAKU 200380-200384, 201398, 201435, 201437) and a large specimen (FAKU 200470, $144.0 \mathrm{~mm} \mathrm{SL}$ ) lacked prickle-like scales on the head, being rather similar to Malacocottus gibber Sakamoto, 1930. Notwithstanding this, the large specimen had six gill rakers, falling within the range of M. zonurus [4-9 in M. zonurus vs. 9-15 in M. gibber (see Shinohara et al. 1992)]. Kitagawa et al. (2008) reported Malacocottus sp. from the Pacific Ocean off the northern Honshu, Japan, characterized by a soft blackish body and no spines on the head. Although the small specimens listed above also had a soft and blackish body, they different from Malacocottus sp. in the preopercular spine character.

\section{Family Agonidae}

40. Aspidophoroides monopterygius (Bloch, 1786) [Japanese name: Tate-tokubire] (Plate V, Fig. 6)

FAKU 200560, 145.7 mm SL, E4, 24 Apr. 2013; FAKU 200700, 171.0 mm SL, B3, 22 Apr. 2013; FAKU 201359, 201360, 149.4-153.2 mm SL, A5, 18 Apr. 2014; FAKU 201392, 201393, 153.6-156.0 mm SL, B3, 19 Apr. 2014.

Remarks: Although Kanayama (1991) synonymized Aspidophoroides bartoni Gilbert, 1896 under A. monopterygius, Sheiko and Mecklenburg (2004) considered them to represent two valid species. Kanayama (1991) and Nakabo and Kai (2013a) are followed here, although the specimens corresponded to A. bartoni sensu (see Sheiko and Mecklenburg 2004).

41. Freemanichthys thompsoni (Jordan and Gilbert in Jordan and Evermann, 1898) [Japanese name: Yase-tokubire] (Plate V, Fig. 7)

FAKU 200701, 153.0 mm SL, B3, 22 Apr. 2013; FAKU 200736, 155.2 mm SL, D5, 22 Apr. 2013.

42. Leptagonus leptorhynchus (Gilbert, 1896) [Japanese name: Tengu-tokubire] (Plate V, Fig. 8)

FAKU 200561, 127.9 mm SL, E4, 24 Apr. 2013; FAKU 200653, 69.5 mm SL, E3, 23 Apr. 2013; FAKU 200687, 113.3 mm SL, A5, 17 Apr. 2013; FAKU 200698, 200699, 104.1-112.8 mm SL, B3, 22 Apr. 2013; FAKU 200715, 200716, 111.1-129.6 mm SL, A3, 17 Apr. 2013; FAKU 200798, 200799, 86.2-95.0 mm SL, D2, 21 Apr. 2013; FAKU 201412-201420, 52.4-150.7 mm SL, A3(2), 23 Apr. 2014.

43. Percis japonica (Pallas, 1769) [Japanese name: Inugochi] (Plate V, Fig. 9)

FAKU 200591-200593, 204.7-262.5 mm SL, C3, 18 Apr. 2013; FAKU 201381, $113.1 \mathrm{~mm} \mathrm{SL,} \mathrm{B4,} 19$ Apr. 2014; FAKU 201431, 43.4 mm SL, D1, 21 Apr. 2014; FAKU 201432-201434, 68.2-150.0 mm SL, E1, 21 Apr. 2014.

44. Podothecus sachi (Jordan and Snyder, 1901) [Japanese name: Tokubire] (Plate V, Figs. 10, 11) FAKU 200580, 162.3 mm SL, female, A4, 18 Apr. 2013; FAKU 200686, 324.0 mm SL, male, A5, 17 Apr. 


\section{FISH OF KITAMI-YAMATO BANK, SOUTHERN SEA OF OKHOTSK}

2013.

45. Tilesina gibbosa Schmidt in Jordan and Starks, 1904 [Japanese name: Oni-shachi-uo] (Plate V, Fig. 12) FAKU 200666-200670, 219.7-262.7 mm SL, A2, 17 Apr. 2013.

\section{Family Cyclopteridae}

46. Aptocyclus ventricosus (Pallas, 1769) [Japanese name: Hotei-uo] (Pate VI, Fig. 1)

FAKU 201402, 209.4 mm SL, G1, 21 Apr. 2014.

47. Eumicrotremus asperrimus (Tanaka, 1912) [Japanese name: Konpeitō] (Plate VI, Figs. 2, 3)

FAKU 200602-200605, 39.9-88.1 mm SL, A1, 17 Apr. 2013; FAKU 200678-200680, 52.3-71.2 mm SL, A2, 17 Apr. 2013; FAKU 200707, 21.1 mm SL, A3, 17 Apr. 2013; FAKU 200733-200735, 23.2-53.2 mm SL, D5, 22 Apr. 2013; FAKU 201384-201387, 19.2-25.8 mm SL, A2, 16 Apr. 2014; FAKU 201388, 49.9 mm SL, B2, 18 Apr. 2014; FAKU 201389, eggs protected by FAKU 201388, B2, 18 Apr. 2014; FAKU 201442, 22.7 mm SL, C1, 20 Apr. 2014.

Remarks: Although E. asperrimus has been characterized as having many bony tubercles over the entire body and an indistinct spinous dorsal fin (Nakabo and Kai 2013b), some of the specimens examined here had few or no bony tubercles and a relatively distinct spinous dorsal fin, rather similar to Cyclopteropsis lindbergi Soldatov, 1930 or Cyclopteropsis bergi Popov, 1929. However, Kai et al. (2015) recently indicated that such differences in E. asperrimus can be attributed to sexual dimorphism, following their molecular and morphological analysis. Accordingly, the present specimens with few bony tubercles and a relatively distinct spinous dorsal fin were identified as that species.

\section{Family Liparidae}

48. Careproctus colletti Gilbert, 1896 [Japanese name: Arasuka-bikunin] (Plate VI, Fig. 4)

FAKU 200528, 200529, 233.0-250.4 mm SL, G2, 20 Apr. 2013; FAKU 200743, $250.1 \mathrm{~mm}$ SL, G1, 19 Apr. 2013.

49. Careproctus cyclocephalus Kido, 1983 [Japanese name: Daruma-kon'nyaku-uo] (Plate VI, Fig. 5)

FAKU 200525, 284.4 mm SL, G2, 20 Apr. 2013.

50. Careproctus furcellus Gilbert and Burke, 1912 [Japanese name: Oguro-kon'nyaku-uo] (Plate VI, Fig. 6)

FAKU 200479*, 200480, 258.0-261.2 mm SL, G2, 20 Apr. 2013; FAKU 200744, 200745, 276.2-355.0 mm SL, G1, 19 Apr. 2013.

51. Careproctus macrodiscus Schmidt, 1950 [Japanese name: Ohōtsuku-kon'nyaku-uo] (Plate VI, Fig. 7)

FAKU 200526, 200527, 207.2-279.4 mm SL, G2, 20 Apr. 2013.

52. Careproctus marginatus Kido, 1988 [Japanese name: Hireguro-bikunin] (Plate VI, Fig. 8)

FAKU 200477, 200478, 188.3-204.0 mm SL, G2, 20 Apr. 2013; FAKU 200742, 191.8 mm SL, G1, 9 Apr. 2013.

53. Careproctus rastrinus species complex [Japanese name: Sake-bikunin species complex] (Plate VI, Fig. 9)

FAKU 200394, 84.2 mm SL, C1, 19 Apr. 2013; FAKU 200403 (caudal part damaged), 200404-200416, 82.6-192.2 mm SL, E1, 19 Apr. 2013; FAKU 200545, 230.8 mm SL, E5, 21 Apr. 2013; FAKU 200746- 
200748, 105.0-252.5 mm SL, C5, 19 Apr. 2013.

Remarks: Kido (1988) indicated that $C$. rastrinus and a closely related species Careproctus trachysoma Gilbert and Burke, 1912, were distinguishable by the body coloration. However, Kai et al.'s (2011) molecular and morphological analyses showed that such differences represented intraspecific variations, C. rastrinus and closely related species ( $=C$. rastrinus species complex) being defined by geographic regions, such as the Sea of Japan, the Sea of Okhotsk, the Pacific coast of Japan, and the Bering Sea. The species examined appeared to be conspecific with "OKH1" of Kai et al. (2011), judging from the collection locality.

54. Careproctus roseofuscus Gilbert and Burke, 1912 [Japanese name: Tobi-bikunin] (Plate VII, Fig. 1)

FAKU 200475, 200476, 76.1-237.6 mm SL, G2, 20 Apr. 2013; FAKU 200516, 242.8 mm SL, E5, 21 Apr. 2013; FAKU 200554, 200555, 254.8-276.9 mm SL, G4, 23 Apr. 2013.

55. Careproctus segaliensis Gilbert and Burke, 1912 [Japanese name: Karafuto-bikunin] (Plate VII, Fig. 2)

FAKU 200556, 75.0 mm SL, G4, 23 Apr. 2013.

Remarks: Reports of this species from the Sea of Japan (Takegawa and Morino 1970; Ueno 1971) are inconclusive since they failed to include voucher specimens or supporting morphological evidence. Lindberg and Krasyukova (1987) reported the species from the east coast of Sakhalin Island, Sea of Okhotsk, nothing a specimen deposited in the Zoological Institute of the Russian Academy of Sciences, St. Petersburg, Russia (ZIN 26321). However, their description was apparently based on the original description of the species, the only confirmed record therefore being that of the holotype (Kido 1988; Sakurai and Shinohara 2008). The specimen listed here as $C$. segaliensis was characterized as follows: trilobed teeth; dorsal-fin rays 63 (61 in holotype, USNM 73336); anal-fin rays 55 (53); pectoral-fin rays 24 (23); caudal-fin rays 6 (6); vertebrae 69 (67); branchiostegal rays 6 ; nasal pores 2 , maxillary pores 5 , preoperculomandibular pores 6 , suprabranchial pores 1 (cephalic pore pattern 2-5-6-1).

56. Careproctus simus Gilbert, 1896 [Japanese name: Tengu-kon'nyaku-uo] (Plate VII, Fig. 3)

FAKU 201401, 165.2 mm SL, G1, 21 Apr. 2014.

Remarks: This species is common in the Bering Sea (Mecklenburg et al. 2002), but only four specimens have previously been recorded from the Sea of Okhotsk (Tsutsui and Amaoka 1997).

57. Careproctus sp. (Plate VII, Fig. 4)

FAKU 201379, 41.0 mm SL, B4, 19 Apr. 2014.

Remarks: The genus Careproctus has been diagnosed by the following characters: pelvic disc present, nostril single, pectoral-fin rays typically less than anal-fin rays, pseudobranch absent, body color non-variegated (Kido 1988; Stein et al. 2001). However, Orr and Maslenikov (2007) emended the diagnosis to include the variegated color pattern. Accordingly, the present specimen was identified as a species of Careproctus sensu Orr and Maslenikov (2007), combining the diagnostic characters of Kido (1988) and Stein et al. (2001) with a variegated color pattern (very thin reddish body with white mottles). Only two Careproctus species with a variegated color pattern have been reported from the Bering Sea, viz. Careproctus comus Orr and Maslenikov, 2007 and Careproctus faunus Orr and Maslenikov, 2007. However, the present specimen differed from both, having 46 dorsal- and 40 anal -fin rays [vs. 50-56, 44-50 in C. comus and 47-51, 41-45 in C. faunus (see Orr and Maslenikov 2007)]. On the other hand, it resemble Temnocora candida (Gilbert and Burke, 1912), known from the Bering Sea and the Gulf of Alaska, in having trilobed teeth, the pupil reduced to a horizontal slit and a very thin reddish body with white mottles (fresh condition) (Gilbert and Burke 1912a; Orr and Maslenikov 2007), in addition to falling within the ranges of that species' dorsaland anal-fin ray counts (43-48 and 36-41, respectively) (Mecklenburg et al. 2002; Orr and Maslenikov 2007). However, genus Temnocora is diagnosed by a lobed dorsal-fin (Burke 1930), which did not occur in the present specimen. Accordingly, we tentatively treated it as a member of genus Careproctus. 
58. Crystallichthys matsushimae (Jordan and Snyder, 1902) [Japanese name: Abachan] (Plate VII, Fig. 5)

FAKU200391, 200392, 146.4 mm SL, C3, 18 Apr. 2013; FAKU 200396-200399, 140.4-260.0 mm SL, C1, 19 Apr. 2013; FAKU 200400, 91.6 mm SL, A1, 17 Apr. 2013; FAKU 200417, 200418, 190.8-258.8 mm SL, C5, 19 Apr. 2013; FAKU 200425-200429, 171.2-277.3 mm SL, C4, 20 Apr. 2013; FAKU 200471, 288.4 mm SL, E2, 20 Apr. 2013; FAKU 200517-200519, 226.8-249.7 mm SL, E5, 21 Apr. 2013; FAKU 200520, 200521, 188.6-228.8 mm SL, D1, 21 Apr. 2013; FAKU 200553, 97.4 mm SL, G4, 23 Apr. 2013; FAKU 200656, 200657, 257.8-316.6 mm SL, E3, 23 Apr. 2013; FAKU 200658, 200659, 278.8-285.1 mm SL, D3, 21 Apr. 2013; FAKU 200737, 255.5 mm SL, B1, 18 Apr. 2013; FAKU 200795, 51.6 mm SL, D3, 21 Apr. 2013; FAKU 201376, 142.4 mm SL, B2, 18 Apr. 2014; FAKU 201404, 201406, 201407, 73.9-154.6 mm SL, B3, 19 Apr. 2014; FAKU 201410, 201411, 104.9-131.7 mm SL, A3(2), 23 Apr. 2014.

Remarks: Crystallichthys matsushimae was recently found to include two color variants, a "red" morphotype, conforming to the earlier descriptions of Gilbert and Burke (1912b) and Kido (1988) and distributed in the northern Sea of Japan, southern Sea of Okhotsk and in the Pacific coast of northern Japan, and a "yellow" morphotype, restricted to the southern Sea of Japan (Tohkairin et al. 2014). All of the specimens examined here conformed to the former.

59. Liparis ochotensis Schmidt, 1904 [Japanese name: Isago-bikunin] (Plate VII, Fig. 6)

FAKU 200390, 280.5mm SL, C3, 18 Apr. 2013; FAKU 200395, 152.9 mm SL, C1, 19 Apr. 2013; FAKU 200423, 200424, 63.6-207.2 mm SL, C2, 20 Apr. 2013; FAKU 200456, 268.6 mm SL, C4, 20 Apr. 2013; FAKU 200457-200460, 199.6-297.5 mm SL, A2, 17 Apr. 2013; FAKU 200472, 200473, 221.5-261.3 mm SL, E2, 20 Apr. 2013; FAKU 200522, 211.9 mm SL, D1, 21 Apr. 2013; FAKU 200540, 200541, 215.9-234.5 mm SL, A1, 17 Apr. 2013; FAKU 200543, 200544, 256.7-282.5 mm SL, E5, 21 Apr. 2013; FAKU 200550, 200551, 209.3-275.9 mm SL, B2, 18 Apr. 2013; FAKU 200552, 311.7 mm SL, G4, 23 Apr. 2013; FAKU 200652, 274.0 mm SL, D4, 23 Apr. 2013; FAKU 200654, 200655, 215.7-229.0 mm SL, E3, 23 Apr. 2013; FAKU 200660, 216.6 mm SL, D3, 21 Apr. 2013; FAKU 200749, 240.3 mm SL, C5, 19 Apr. 2013; FAKU 200750-200752, 236.2-285.7 mm SL, E1, 19 Apt. 2013; FAKU 201363-201365, 109.4-216.1 mm SL, A1, 17 Apr. 2014.

60. Liparis tessellatus (Gilbert and Burke, 1912) [Japanese name: Bikunin] (Plate VII, Fig. 7)

FAKU 200559, 82.9 mm SL, E4, 24 Apr. 2013.

61. Paraliparis grandis Schmidt, 1950 [Japanese name: Hira-inkiuo] (Plate VII, Fig. 8)

FAKU 200481, 309.1 mm SL, G2, 20 Apr. 2013.

\section{Paraliparis sp. (Plate VII, Fig. 9)}

FAKU 200474, 85.7 mm SL, G2, 20 Apr. 2013.

Remarks: The specimen was identified as a species of Paraliparis due to the absence of several characters, including a pseudobranch, coronal pore, barbels or skin flaps and modified pelvic sucking disk, found in most other liparid genera (Kido 1988; Stein et al. 2001). Although most Paraliparis species have a single suprabranchial pore, the specimen examined here had two such pores, reminiscent of Paraliparis pectoralis Stein, 1978 (see Kido 1984, 1993; Mecklenburg et al. 2002). However, it differed from the latter in dorsaland anal-fin ray counts (50 vs. 55-58; 45 vs. 49-51, respectively) (Kido 1984). These differences may suggest that the present specimen represents an undescribed species, but we were unable to name it without more specimens.

\section{Family Zoarcidae}

63. Bothrocara hollandi (Jordan and Hubbs, 1925) [Japanese name: Noro-genge] (Plate VIII, Fig. 1) 
FAKU 200463-200465, 170.2-226.1 mm SL, G1, 19 Apr. 2013.

64. Bothrocara zestum Jordan and Fowler, 1902 [Japanese name: Shiro-genge] (Plate VIII, Fig. 2) FAKU 200785, caudal part damaged, G2, 20 Apr. 2013.

65. Bothrocarina microcephala (Schmidt, 1938) [Japanese name: Kamuchakka-genge] (Plate VIII, Fig. 3) FAKU 200373, 200374, 200462, 110.5-238.1 mm SL, G1, 19 Apr. 2013; FAKU 200784, 137.8 mm SL, G2, 20 Apr. 2013.

66. Gymnelopsis ochotensis (Popov, 1931) [Japanese name: Ohōtsuku-genge] (Plate VIII, Fig. 4) FAKU 200393, 169.2 mm SL, C1, 19 Apr. 2013; FAKU 201443, 179.4 mm SL, C1, 20 Apr. 2014.

67. Lycodapus microchir Schmidt, 1950 [Japanese name: Yawara-genge] (Plate VIII, Fig. 5)

FAKU 200786-200789, 121.0-135.0 mm SL, G2, 20 Apr. 2013.

68. Lycodes albonotatus (Taranetz and Andriashev, 1934) [Japanese name: Hana-genge] (Plate VIII, Fig. 6) FAKU 200466, 200467, 192.7-230.3 mm SL, G1, 19 Apr. 2013.

69. Lycodes matsubarai Toyoshima, 1985 [Japanese name: Matsubara-genge] (Plate VIII, Fig. 7)

FAKU 200546, 200547, 200.3-213.8 mm SL, E5, 21 Apr. 2013; FAKU 200581, 265.2 mm SL, A1, 17 Apr. 2013; FAKU 201367, 201370-201374, 180.7-242.1 mm SL, A1, 17 Apr. 2014; FAKU 201441, 162.0 mm SL, C1, 20 Apr. 2014.

70. Lycodes microporus Toyoshima in Amaoka et al., 1983 [Japanese name: Yase-mayugaji] (Plate VIII, Fig. 8)

FAKU 200372, 157.8 mm SL, G1, 19 Apr. 2013; FAKU 200783, 133.7 mm SL, G2, 20 Apr. 2013.

Remarks: This species was redescribed and considered rare by Ikeda et al. (2008). The present specimens were identified by the following characters: sensory pores on head tubular; lateral line single, ventral; body and head blackish-brown, without blotches.

71. Lycodes pectoralis Toyoshima, 1985 [Japanese name: Kitano-kurogenge] (Plate VIII, Fig. 9)

FAKU 200563-200569, 220.3-275.4 mm SL, E1, 19 Apr. 2013.

72. Lycodes tanakae Jordan and Thompson, 1914 [Japanese name: Tanaka-genge] (Plate VIII, Fig. 10)

FAKU 200422, 213.4 mm SL, C2, 20 Apr. 2013; FAKU 201368, 201369, 178.0-262.1 mm SL, A1, 17 Apr. 2014.

73. Petroschmidtia teraoi (Katayama, 1943) [Japanese name: Hinagenge] (Plate VIII, Fig. 11)

FAKU 201444-201447, 97.9-109.0 mm SL, C1, 20 Apr. 2014.

Remarks: Although this species has been recognized as a member of Lycodes (e.g., Anderson and Fedorov 2004; Hatooka 2013b), Nazarkin et al. (2014) removed it to the genus Petroschmidtia. The species was first reported from the Sea of Okhotsk by Nazarkin et al. (2014).

\section{Family Stichaeidae}

74. Anisarchus medius (Reinhardt, 1837) [Japanese name: Nise-medamaginpo] (Plate IX, Fig. 1)

FAKU 200584 (caudal part damaged), 200585, 131.6 mm SL, A1, 17 Apr. 2013; FAKU 201366, 201371, 159.1-159.8 mm SL, A1, 17 Apr. 2014. 
Remarks: Yamanaka and Yabe (2012) recently redescribed this species on the basis of specimens collected from the Sea of Okhotsk, the first reliable records from waters off Japan.

75. Leptoclinus maculatus (Fries, 1838) [Japanese name: Yase-ginpo] (Plate IX, Fig. 2)

FAKU 200524, 159.3 mm SL, D1, 21 Apr. 2013; FAKU 200582, 200583, 200586*, 200587*, 200588*, 200589*, 110.3-177.1 mm SL, A1, 17 Apr. 2013; FAKU 200801, 162.2 mm SL, D4, 23 Apr. 2013.

Remarks: Miyahara et al.'s (2005) record of Leptoclinus maculatus diaphanocarus (Schmidt, 1904), based on a specimen collected from the Sea of Okhotsk, was later referred to L. maculatus by Hatooka (2013a). And Mecklenburg et al. (2011) showed that L. m. diaphanocarus and L. m. maculatus were indistinguishable on the basis of mitochondrial COI sequence analyses.

76. Lumpenella longirostris (Evermann and Goldsborough, 1907) [Japanese name: Nezumi-ginpo] (Plate IX, Fig. 3)

FAKU 200375-200377, 200468, 200469, 117.7-332.0 mm SL, G1, 19 Apr. 2013.

77. Stichaeopsis epallax (Jordan and Snyder, 1902) [Japanese name: Ame-gaji] (Plate IX, Fig. 4)

FAKU 201390, 201391, 144.3-178.9 mm SL, B3, 19 Apr. 2014.

78. Stichaeus grigorjewi Herzenstein, 1890 [Japanese name: Nagaduka] (Plate IX, Fig. 5)

FAKU 201378, 109.7 mm SL, A4, 18 Apr. 2014.

79. Stichaeus nozawae Jordan and Snyder, 1902 [Japanese name: Tauegaji] (Plate IX, Fig. 6)

FAKU 200523, 217.7 mm SL, D1, 21 Apr. 2013; FAKU 200665, 224.3 mm SL, A2, 17 Apr. 2013; FAKU 200696, 200697, 105.9-119.0 mm SL, B5, 22 Apr. 2013; FAKU 200708-200711, 121.5-164.2 mm SL, A3, 17 Apr. 2013.

80. Stichaeus ochriamkini Taranetz, 1935 [Japanese name: Kita-tauegaji] (Plate IX, Fig. 7)

FAKU 201356, 201357, 114.8-130.6 mm SL, A5, 18 Apr. 2014.

\section{Order Pleuronectiformes \\ Family Pleuronectidae}

81. Acanthopsetta nadeshnyi Schmidt, 1904 [Japanese name: Urokome-garei] (Plate IX, Fig. 8)

FAKU 200692-200694, 135.2-162.4 mm SL, A2, 17 Apr. 2013; FAKU 200723-200728, 126.9-202.3 mm SL, A3, 17 Apr. 2013; FAKU 200804-200806, 154.3-181.8 mm SL, D4, 23 Apr. 2013; FAKU 200826, 246.6 mm SL, B1, 18 Apr. 2013.

82. Atheresthes evermanni Jordan and Starks, 1904 [Japanese name: Abura-garei] (Plate IX, Fig. 9)

FAKU 200558, 330.0 mm SL, E1, 19 Apr. 2013; FAKU 200807, 301.0 mm SL, D4, 23 Apr. 2013.

83. Clidoderma asperrimum (Temminck and Schlegel, 1846) [Japanese name: Same-garei] (Plate IX, Fig. 10)

FAKU 200761, 261.3 mm SL, B4, 24 Apr. 2013.

84. Glyptocephalus stelleri (Schmidt, 1904) [Japanese name: Hireguro] (Plate IX, Fig. 11)

FAKU 200530-200532, 84.7-127.8 mm SL, D1, 21 Apr. 2013; FAKU 200767, 245.3 mm SL, B1, 18 Apr. 2013; FAKU 200777-200779, 132.6-168.6 mm SL, A1, 17 Apr. 2013. 
85. Hippoglossoides dubius Schmidt, 1904 [Japanese name: Aka-garei] (Plate X, Fig. 1)

FAKU 200533-200537, 122.0-165.7 mm SL, D1, 21 Apr. 2013; FAKU 200720, $210.3 \mathrm{~mm}$ SL, A3, 17 Apr. 2013; FAKU 200763-200766, 192.1-317.0 mm SL, B1, 18 Apr. 2013; FAKU 200774-200776, 128.7204.5 mm SL, A1, 17 Apr. 2013; FAKU 200821-200824, 171.1-204.0 mm SL, B1, 18 Apr. 2013.

86. Hippoglossoides pinetorum (Jordan and Starks, 1904) [Japanese name: Sōhachi] (Plate X, Fig. 2)

FAKU 200684, 200685, 163.0-166.6 mm SL, A5, 17 Apr. 2013; FAKU 200721, 200722, 213.7-226.2 mm SL, A3, 17 Apr. 2013; FAKU 200803, 172.3 mm SL, D4, 23 Apr. 2013; FAKU 200825, 183.9 mm SL, B1, 18 Apr. 2013.

87. Hippoglossus stenolepis Schmidt, 1904 [Japanese name: Ohyō] (Plate X, Fig. 3)

FAKU 200461, 389.0 mm SL, G1, 19 Apr. 2013.

88. Pleuronectes asper Pallas, 1814 [Japanese name: Kogane-garei] (Plate X, Fig. 4)

FAKU 200695, 269.1 mm SL, B5, 21 Apr. 2013; FAKU 200773, 267.7 mm SL, A1, 17 Apr. 2013.

89. Pleuronectes herzensteini (Jordan and Snyder, 1901) [Japanese name: Ma-garei] (Plate X, Fig. 5)

FAKU 200760, 185.5 mm SL, B4, 24 Apr. 2013; FAKU 201361, 186.3 mm SL, A3, 17 Apr. 2014.

90. Pleuronectes mochigarei (Snyder, 1911) [Japanese name: Asaba-garei] (Plate X, Fig. 6)

FAKU 200677, 228.6 mm SL, G4, 23 Apr. 2013; FAKU 200717, 200718, 200719*, 221.9-245.2 mm SL, A3, 17 Apr. 2013; FAKU 200762, 252.8 mm SL, B4, 24 Apr. 2013; FAKU 200768-200772, 243.0-270.6 mm SL, B1, 18 Apr. 2013; FAKU 200802, 237.9 mm SL, D4, 23 Apr. 2013; FAKU 200827-200832, 236.6-312.0 mm SL, B1, 18 Apr. 2013.

91. Pleuronectes sakhalinensis (Hubbs, 1915) [Japanese name: Karafuto-garei] (Plate X, Fig. 7)

FAKU 200780-200782, 155.4-178.6 mm SL, A1, 17 Apr. 2013.

92. Reinhardtius hippoglossoides (Walbaum, 1792) [Japanese name: Karasu-garei] (Plate X, Fig. 8)

FAKU 200402, 111.4 mm SL, A1, 17 Apr. 2013; FAKU 200690, 200691, 106.5-128.1 mm SL, A2, 17 Apr. 2013; FAKU 200729, 200730, 122.3-123.4 mm SL, D5, 22 Apr. 2013.

\section{Concluding remarks}

Although comprehensive checklists of fishes have been published for the Sea of Japan (Shinohara et al. 2014), the Pacific coast of Japan (Shinohara et al. 2009), and the Bering Sea (Mecklenburg et al. 2002), reliable checklists focused on the Sea of Okhotsk, in particular the southern region, are limited. In this report, a total of 450 specimens from the western part of the Kitami-Yamato Bank, southern Sea of Okhotsk, were classified into 92 species and 21 families. Although most families in this report are represented by 1-3 species, Cottidae, Liparidae, Zoarcidae, and Pleuronectidae included 13, 15, 11 and 12 species, respectively, comprising more than half of the total number of species. Furthermore, three of the latter four families are mostly secondary deepwater fishes (Schmidt 1950; Nishimura 1974), which group characterizes the ichthyofauna of the Sea of Okhotsk (Schmidt 1950).

Amaoka et al. (1983) reported a total of 80 species [correctly 79 spp., Bathyraja caeluronigricans Ishiyama and Ishihara, 1977 and Bathyraja notoroensis Ishiyama and Ishihara, 1977 being synonymous under Bathyraja matsubarai (Ishiyama, 1952) (see Hatooka et al. 2013)], belonging to 25 families from the eastern 
area of Kitami-Yamato Bank. Although 16 families are common to the present report and Amaoka et al. (1983), the latter included many primary deepwater fishes (Schmidt 1950; Nishimura 1974), numbering 26 species in 12 families (e.g., family Macrouridae). The present report, however, includes only four species representing primary deepwater families (Microstomatidae, Myctophidae and Moridae), primarily due to the difference in collection site depths [ca. 120-520 m depth (present report), 400-1400 m depth (Amaoka et al. 1983)].

Nishimura's (1983) suggestion that the secondary deepwater fishes in the Sea of Japan originated from the Sea of Okhotsk is partly supported by comparisons of secondary deepwater fishes, both here and in previous studies conducted in the Sea of Japan (Shinohara et al. 2014: 363 species) and on the Pacific coast off northern Honshu, Japan (Shinohara et al. 2009, 496 species). Eleven of 13 cottid species are common to the Sea of Okhotsk and Sea of Japan, but only three to the former and the Pacific coast. Similarly, five of 11 zoarcid species are common to the Sea of Okhotsk and Sea of Japan, but only three to the former and the Pacific coast (Shinohara et al. 2009). However, seven liparid species were recorded both here and by Shinohara et al. (2009) (Pacific coast of northern Japan), and five species both here and by Shinohara et al. (2014) (Sea of Japan).

More comprehensive surveys in the southern Sea of Okhotsk should further disclose the relationships of the ichthyofauna with neighboring waters, in addition to more extensive biogeographic insights across the North Pacific.

\section{Acknowledgments}

We are very grateful to officers and crews of R/V Kaiyo-maru No. 5 (Ocean Engineering Co. LTD., Tokyo, Japan) for their help in collecting specimens. We also thank G.S. Hardy for his critical reading of the manuscript, and Mr. R. Misawa (Kochi University) for helpful comments. This study was supported in part by a grant-in-aid for scientific research (B) from JSPS (24370036) and the Fisheries Agency of Japan under the projects of 'Assessment of Fisheries Stocks in the Waters around Japan'. This study is dedicated to late Shouichi Tabata, the former captain of R/V Kaiyo-maru No. 5.

\section{Literature cited}

Amaoka, K., Nakaya, K., Shintani, H. and Yasui, T. 1983. Fishes from the northeastern Sea of Japan and the Okhotsk Sea of Hokkaido. Japan Fisheries Resource Conservation Association, Tokyo, 327pp. (In Japanese and English).

Anderson, M. E. and Fedorov, V. V. 2004. Family Zoarcidae Swainson 1839, eelpouts. California Academy of Sciences Annotated Checklists of Fishes, 34, 1-58.

Bezverkhniy, V. L., Pletnev, S. P. and Nabiullin A. A. 2002. Outline of the geological structure and development of the Kuril Island System and adjacent regions. Flora and fauna of the Kuril Islands materials of the International Kuril Island Project, 35-66.

Briggs, J. C. and Bowen, B. W. 2012. A realignment of marine biogeographic province with particular reference to fish distributions. Journal of Biogeography, 39, 12-30.

Burke, V. 1930. Revision of the fishes of the family Liparidae. Bulletin of the U.S. National Museum, 150, $1-204$.

Coulson, M. W., Marshall, H. D., Pepin, P. and Carr, S. M. 2006. Mitochondrial genomics of gadine fishes: implications for taxonomy and biogeographic origins from whole-genome data sets. Genome, 49, 11151130.

Ebert, D. A., White, W. T., Ho, H., Last, P. R., Nakaya, K., Séret, B., Straube, N., Naylor, G. P. and Carvalho, 
M. R. 2013. An annotated checklist of the chondrichthyans of Taiwan. Zootaxa, 3752, 279-386.

Fedorov, V. V., Chereshnev, I. A., Nazarkin, M. V., Shestakov, A. V. and Volobue, V. V. 2003. Catalogue of marine and freshwater fish from the northern part of the Sea of Okhotsk. Dal'nauka, Vladivostok. 202 pp. (In Russian).

Gilbert, C. H. and Burke, C. V. 1912a. Fishes from Bering Sea and Kamchatka. Bulletin of the Bureau of Fishes, 30, 31-96.

Gilbert, C. H. and Burke, C. V. 1912b. New cyclogasterid fishes from Japan. Proceeding of the United States National Museum, 42, 351-380, pls. 41-48.

Gorbarenko, S. A., Khusid, T. A., Basov, I. A., Oba, T., Southon, J. R. and Koizumi, I. 2002. Glacial Holocene environment of the southeastern Okhotsk Sea: evidence from geochemical and palaeontological data. Palaeogeography, Palaeoclimatology, Palaeoecology, 177, 237-263.

Harada, N., Ahagon, N., Sakamoto, T., Uchida, M., Ikehara, M. and Shibata, Y. 2006. Rapid fluctuation of alkenone temperature in the southern Okhotsk Sea during the past $120 \mathrm{ky}$. Global and Planetary Change, $53,29-46$.

Hatooka, H. 2013a. Stichaeidae. In, Nakabo, T. (ed.), Fishes of Japan with pictorial keys to the species. Tokai University Press, Hadano, pp. 1239-1251, 2082-2086 (In Japanese).

Hatooka, H. 2013b. Zoarcidae. In, Nakabo, T. (ed.), Fishes of Japan with pictorial keys to the species. Tokai University Press, Hadano, pp. 1220-1237, 2076-2082 (In Japanese).

Hatooka, H., Yamada, U., Aizawa, M., Yamaguchi, A. and Yagishita, N. 2013. Rajidae. In, Nakabo, T. (ed.), Fishes of Japan with pictorial keys to the species. Tokai University Press, Hadano, pp. 205-216, 17711773 (In Japanese).

Higuchi, M. and Goto, A. 1996. Genetic structure of threespine stickleback, Gasterosteus aculeatus, in Lake Harutori, Japan, with reference to coexisting anadromous and freshwater forms. Ichthyological Research, 43, 349-358.

Higuchi, M., Sakai, H. and Goto, A. 2014. A new threespine stickleback, Gasterosteus nipponicus sp. nov. (Teleostei: Gasterosteidae), from the Japan Sea region. Ichthyological Research, 61, 341-351.

Hosoya, K. 2000. Gasterosteidae. In, Nakabo, T. (ed.), Fishes of Japan with pictorial keys to the species. Tokai University Press, Hadano, pp. 513-514, 1506-1507 (In Japanese. English translation 2002).

Ikeda, S., Imamura, H. and Nakaya, K. 2008. Redescription of two eelpouts, Lycodes microporus Toyoshima 1983 and Lycodes ocellatus Toyoshima 1985. Ichthyological Research, 55, 356-366.

Ishihara, H., Treloar, M., Bor, P. H. F., Senou, H. and Jeong C. H. 2012. The comparative morphology of skate egg capsules (Chondrichthyes: Elasmobranchii: Rajiformes). Bulletin of the Kanagawa Prefectural Museum Natural Science, 41, 9-25.

Ishiyama, R. 1958. Studies of the rajid fishes (Rajidae) found in the waters around Japan. Journal of the Shimonoseki Callege of Fisheries, 7, 193-394, pls. 1-3.

Kai, Y., Orr, J. W., Sakai, K. and Nakabo, T. 2011. Genetic and morphological evidence for cryptic diversity in the Careproctus rastrinus species complex (Liparidae) of the North Pacific. Ichthyological Research, $58,143-154$.

Kai, Y., Stevenson, D. E., Ueda, Y., Hamatsu, T. and Nakabo, T. 2015. Molecular insights into geographic and morphological variation within the Eumicrotremus asperrimus species complex (Cottoidei: Cyclopteridae). Ichthyological Research, DOI 10.1007/s10228-014-0453-4.

Kanayama, T. 1991. Taxonomy and phylogeny of the family Agonidae (Pisces: Scorpaeniformes). Memoirs of the Faculty of Fisheries Hokkaido University, 38, 1-199.

Kido, K. 1984. Occurrence of the liparidid fish, Paraliparis pectoralis, in the Bering Sea. Japanese Journal of Ichthyology, 32, 203-204.

Kido, K. 1988. Phylogeny of the family Liparidae, with taxonomy of the species found around Japan. Memoirs of the Faculty of Fisheries, Hokkaido University, 35: 125-256.

Kido, K. 1993. New records of Paraliparis pectoralis and P. nanus (Liparidae) from Japan. Japanese 


\section{FISH OF KITAMI-YAMATO BANK, SOUTHERN SEA OF OKHOTSK}

Journal of Ichthyology, 40, 107-109.

Kitagawa, D., Imamura, H., Goto, T., Ishito, Y., Fujiwara, K. and Ueda, Y. 2008. Field guide of the fishes from Tohoku District, north-eastern waters of Japan. Tokai University Press, Hadano, xvii + 140pp.

Lindberg, G. U. and Fedorov, V. V. 1993. Fishes of the Sea of Japan and the adjacent areas of the Sea of Okhotsk and the Yellow Sea. Part 6: Teleostomi, Osteichthyes, Actinopterygii. XXXI. Pleuronectiformes (CXCV. Fam. Psettoidae-CCI. Fam. Cynoglossidae). Handbook on the Identification of Animals, Zoological Institute of Russian Academy, 1-271 (In Russian).

Lindberg, G. U., Fedorov, V. V. and Krasyukova, Z. V. 1997. Fishes of the Sea of Japan and the adjacent areas of the Sea of Okhotsk and the Yellow Sea. Part 7: Teleostomi, Actinopterygii, Osteichthyes. XXXII. Dactylopteriformes-XXXVII. Pegasiformes. Gidrometioizdat, St. Petersburg, 350 pp. (In Russian).

Lindberg, G. U. and Krasyukova, Z. V. 1969. Fishes of the Sea of Japan and the adjacent areas of the Sea of Okhotsk and the Yellow Sea. Part 3: Teleostomi. XXIX. Perciformes. 1. Percoidei (XC. Fam. SerranidaeCXLIV. Fam. Champsodontidae). Nauka SSSR, Leningrad, 480pp. (In Russian. English translation,1971).

Lindberg, G. U. and Krasyukova, Z. V. 1975. Fishes of the Sea of Japan and the adjacent areas of the Sea of Okhotsk and the Yellow Sea. Part 4: Teleostomi. XXIX. Perciformes 2. Blennioidei-13. Gobioidei (CXLV. Fam. Anarhichaedidae-CLXXV. Fam. Periophthamidae). Nauka SSSR, Leningrad, 463pp. (In Russian. English translation, 1989).

Lindberg, G. U. and Krasyukova, Z. V. 1987. Fishes of the Sea of Japan and the adjacent areas of the Sea of Okhotsk and the Yellow Sea. Part 5: Teleostomi, Osteichthyes, Actinopterygii. XXX. Scorpaeniformes (CLXXVI. Fam. Scorpaenidae-CXCIV. Fam. Liparididae). Nauka SSSR, Leningrad, 526pp. (In Russian).

Lindberg, G. U. and Legeza, M. I. 1959. Fishes of the Sea of Japan and the adjacent areas of the Sea of Okhotsk and the Yellow Sea. Part 1: Amphioxi. Petromyzones. Myxini. Elasmobranchii. Holocephali. Izdatel'stvo Akademii Nauka SSSR, Moscow-Leningrad, 208pp. (In Russian. English translation, 1967).

Lindberg, G. U. and Legeza, M. I. 1965. Fishes of the Sea of Japan and the adjacent areas of the Sea of Okhotsk and the Yellow Sea, Part 2: Teleostomi. XII. Acipenserformes-XXVIII. Polynemiformes. Izdatel'stvo Akademii Nauk SSSR, Moscow-Leningrad, 391pp. (In Russian. English translation, 1969).

Liu, J. X., Gao, T. X., Wu, S. F. and Zhang, Y. P. 2007. Pleistocene isolation in the Northwestern Pacific marginal seas and limited dispersal in a marine fish, Chelon haematocheilus (Temminck \& Schlegel, 1845). Molecular Ecology, 16, 275-288.

Maeda, K. and Tsutsui, D. 2003. A list of fishes from Hokkaido. In, Ueda, K. Maeda, K., Shimada, H. and Takami, T. (eds.), Fisheries and Aquatic Life in Hokkaido. Hokkaido Shinbunsha, Sapporo, pp. 481-504 (In Japanese).

Mecklenburg, C. W., Mecklenburg, T. A. and Thorsteinson, L. K. 2002. Fishes of Alaska. American Fisheries Society, Bethesda, Maryland, xxxvii + 1037pp.

Mecklenburg C. W., Møller, P. R. and Steinke, D. 2011. Biodiversity of arctic marine fishes: taxonomy and zoogeography. Marine Biodiversity, 41, 109-140.

Miyahara, H., Muto, M. and Yabe, M. 2005. First record of Leptoclinus maculatus diaphanocarus (Stichaeidae) collected from Hokkaido. Japanese Journal of Ichthyology, 52, 27-30 (In Japanese).

Nakabo, T. (ed.) 2013. Fishes of Japan with pictorial keys to the species, third edition. Tokai University Press, Hadano, xlix + 2428pp. (In Japanese).

Nakabo, T. and Kai, Y. 2013a. Agonidae. In, Nakabo, T. (ed.), Fishes of Japan with pictorial keys to the species. Tokai University Press, Hadano, pp. 1193-1201, 2069-2071 (In Japanese).

Nakabo, T. and Kai, Y. 2013b. Cyclopteridae. In, Nakabo, T. (ed.), Fishes of Japan with pictorial keys to the species. Tokai University Press, Hadano, pp. 1202-1204, 2071, 2072 (In Japanese).

Nakabo, T. and Kai, Y. 2013c. Gadidae. In, Nakabo, T. (ed.), Fishes of Japan with pictorial keys to the species. Tokai University Press, Hadano, pp. 489-490, 1870 (In Japanese).

Nakabo, T. and Kai, Y. 2013d. Psychrolutidae. In, Nakabo, T. (ed.), Fishes of Japan with pictorial keys to the species. Tokai University Press, Hadano, pp. 1189-1192, 2067-2069 (In Japanese). 
Nazarkin, M. V., Shinohara, G. and Shirai, S. M. 2014. Phylogeny and taxonomy of Petroschmidtia teraoi (Katayama, 1943) (Osteichthyes: Perciformes: Zoarcidae). Zootaxa, 3780: 171-193.

Nishimura, S. 1974. Origin of the Sea of Japan. Tsukiji-shokan, Tokyo, iii + 227pp. (In Japanese).

Nishimura, S. 1983. Okhotsk Sea, Japan Sea, East China Sea. In, Ketchum, B. K. (ed.), Ecosystems of the world, vol. 26, estuaries and enclosed seas. Elsevier, Amsterdam, pp. 375-401.

Orr, J. W. and Maslenikov, K. P. 2007. Two new variegated snailfishes of the genus Careproctus (Teleostei: Scorpaeniformes: Liparidae) from the Aleutian Islands, Alaska. Copeia, 2007, 699-710.

Page, L. M., Espinosa-Pérez, H., Findley, L. T., Gilbert, C. R., Lea, R. N., Mandrak, N. E., Mayden, R. L. and Nelson, J. S. 2013. Common and scientific names of fishes from the United States, Canada, and Mexico, 7th edition. American Fisheries Society Special Publication 34, 243pp.

Parin, N. V. 2001. An annotated catalogue of fish-like vertebrates and fishes of the Sea of Russia and adjacent countries: Part 1. Orders Myxiniformes-Gasterosteiformes. Journal of Ichthyology, 41(Supply 1), S60S135.

Parin, N. V. 2003. An annotated catalogue of fish-like vertebrates and fishes of the Sea of Russia and adjacent countries: Part 3. Orders Perciformes (excluding suborder Gobioidei, Zoarcoidei and Stichaeoidei) and Tetraodontiformes. Journal of Ichthyology, 43(Supplement 1), S1-S40.

Parin, N. V., Fedorov, V. V. and Sheiko, B. A. 2002. An annotated catalogue of fish-like vertebrates and fishes of the Sea of Russia and adjacent countries: Part 2. Order Scorpaeniformes. Journal of Ichthyology, 42(Supplement 1), S660-S135.

Sakurai, H. and Shinohara, G. 2008. Careproctus rotundifrons, a new snailfish (Scorpaeniformes: Liparidae) from Japan. Bulletin of the National Museum Science A, Supplement 2, 39-45.

Schmidt, P. Y. 1950. Fish of the Sea of Okhotsk. Academy of Sciences of USSR, Leningrad. Izdatel'stvo Akademii Nauka SSSR, Moscow-Leningrad, xiv + 392pp. (In Russian. English translation, 1965).

Sheiko, B. A. and Mecklenburg, C. W. 2004. Family Agonidae Swainson 1839, poachers. California Academy of Sciences, Annotated Checklists of Fishes, 30, 1-27.

Shinohara, G., Nakae, M., Ueda, Y., Kojima, S. and Matsuura, K. 2014. Annotated checklist of deep-sea fishes of the Sea of Japan. Deep-sea Fauna of the Sea of Japan, edited by T. Fujita, National Museum of Nature Science Monographs, 44, 225-291.

Shinohara, G., Narimatsu, Y., Hattori, T., Ito, M., Takata, Y. and Matsuura, K. 2009. Annotated checklist of deep-sea from the pacific coast off Tohoku district, Japan. National Museum of Nature and Science Monographs, 39, 683-735.

Shinohara, G., Nazarkin, M. V., Nobetsu, T. and Yabe, M. 2012. A preliminary list of marine fishes found in the Nemuro Strait between Hokkaido and Kunashiri Islands. Bulletin of the National Museum of Nature and Sciences Series A, 38, 181-205.

Shinohara, G. Yabe, M., Amaoka, K. and Meguro, T. 1992. A psychrolutid, Malacocottus gibber, collected from the mesopelagic zone of the Sea of Japan, with comments on its intraspecific variation. Japanese Journal of Ichthyology, 38, 419-424 (In English and Japanese summary).

Soldatov, V. K. and Lindberg, G. J. 1930. A review of the fishes of the Seas of the Far East. Bulletins of the Pacific Scientific Fisheries Institute, xlvii + 576pp., 16pls.

Stein, D. L., Chernova, N. V. and Andriashev, A. P. 2001. Snailfishes (Pisces: Liparidae) of Australia, including descriptions of thirty new species. Record of the Australian Museum, 53, 341-406.

Takegawa, Y. and Morino, H. 1970. Fishes from Wakasa Bay, Japan Sea. Publications of the Seto Marine biological Laboratory, 17, 373-392.

Taranetz, A. Y. 1937. Handbook for identification of fishes of Soviet Far East and adjacent waters. Bulletin of the Pacific Research Institute of Fisheries and Oceanography, 11, 1-200 (In Russian. Japanese translation, 1954).

Tohkairin, A., Kai, Y., Ueda, Y., Hamatsu, T., Ito, M. and Nakabo, T. 2014. Morphological divergence between two color morphotypes of Crystallichthys matsushimae (Cottoidei: Liparidae). Ichthyological 


\section{FISH OF KITAMI-YAMATO BANK, SOUTHERN SEA OF OKHOTSK}

Research, DOI 10.1007/s10228-014-0414-y.

Tsutsui, D. and Amaoka, K. 1997. First record of the snail fish, Careproctus simus (Scorpaeniformes: Liparidae), from Japan. Ichthyological Research, 44, 89-91.

Tyler, P. A. 2002. Deep-sea eukaryote ecology of the semi-isolated basins off Japan. Journal of Oceanography, 58, 333-341.

Ueno, T. 1971. List of the marine fishes from the waters of Hokkaido and its adjacent regions. Scientific Reports of the Hokkaido Experimental Station, 13, 61-102 (In Japanese and English summary).

Wang P. 1999. Response of Western Pacific marginal seas to glacial cycles: paleoceanographic and sedimentological features. Marine Geology, 156, 5-39.

$\mathrm{Xu}, \mathrm{J}$. and Chu, K. H. 2012. Genome scan of the mitten crab Eriocheir sensu stricto in East Asia: population differentiation, hybridization and adaptive speciation. Molecular Phylogenetics and Evolution, 64, 118129.

Yamada, M., Higuchi, M. and Goto, A. 2001. Extensive introgression of mitochondrial DNA found between two genetically divergent forms of threespine stickleback, Gasterosteus aculeatus, around Japan. Environmental Biology of Fishes, 61, 269-284.

Yamanaka, T. and Yabe, M. 2012. Record of Anisarchus medius (Stichaeidae) from Japan. Japanese Journal of Ichthyology, 59, 69-74 (In Japanese).

Received: 30 Nov. 2014

Accepted: 25 Dec. 2014

Published online: 31 Jan. 2015 


\section{TOHKAIRIN, A. ET AL.}
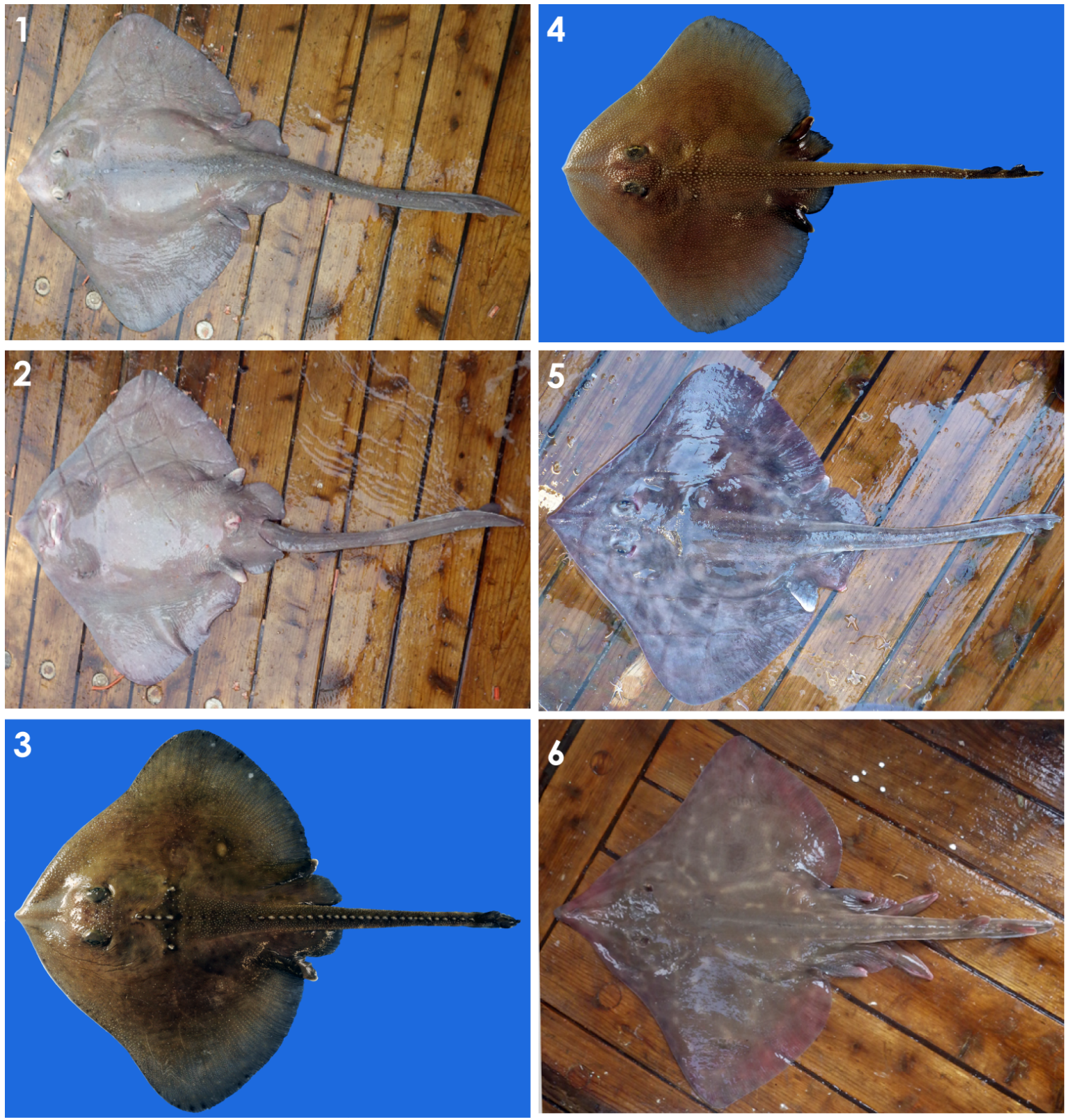

Plate I. Family Rajidae (Figs. 1-6). Fig. 1. Bathyraja matsubarai, dorsal side (FAKU 201463). Fig. 2. Bathyraja matsubarai, ventral side (FAKU 201463). Fig. 3. Bathyraja smirnovi (FAKU 200793, $356.0 \mathrm{~mm}$ TL). Fig. 4. Bathyraja trachouros (FAKU 200676, $279.9 \mathrm{~mm} \mathrm{TL}$ ). Fig. 5. Bathyraja violacea. Fig. 6. Beringraja pulchra (FAKU 201474). 


\section{FISH OF KITAMI-YAMATO BANK, SOUTHERN SEA OF OKHOTSK}
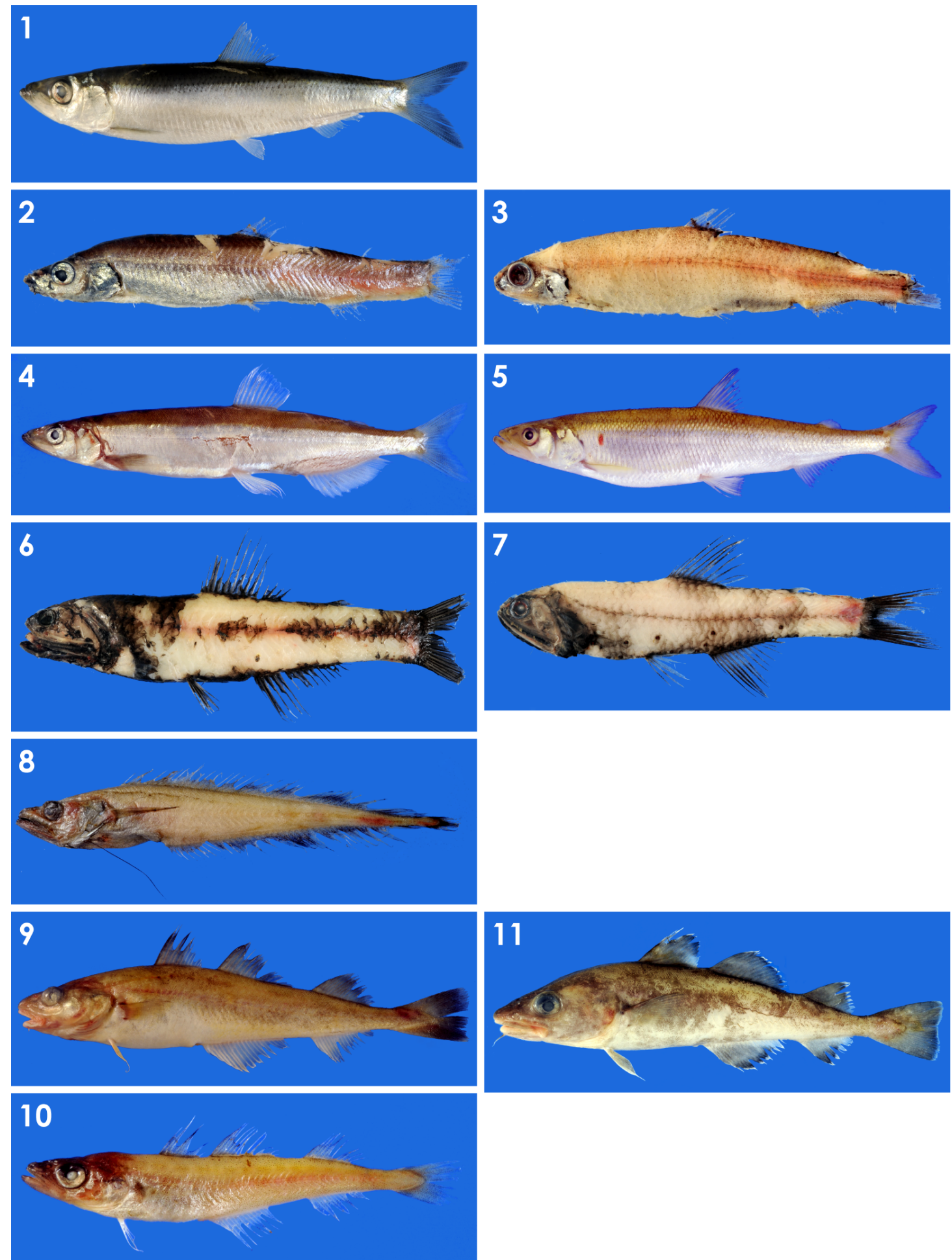

Plate II. Families Clupeidae (Fig. 1), Microstomatidae (Figs. 2, 3), Osmeridae (Figs. 4, 5), Myctophidae (Figs. 6, 7), Moridae (Fig. 8) and Gadidae (Figs. 9-11). Fig. 1. Clupea pallasii (FAKU 200548, 147.5 mm SL). Fig. 2. Leuroglossus schmidti (FAKU 200388, $134.0 \mathrm{~mm} \mathrm{SL).} \mathrm{Fig.} \mathrm{3.} \mathrm{Lipolagus}$ ochotensis (FAKU 200387, $139.6 \mathrm{~mm}$ SL). Fig. 4. Mallotus villosus (FAKU 200596, $152.4 \mathrm{~mm} \mathrm{SL}$ ). Fig. 5. Osmerus dentex (FAKU 200421, $189.6 \mathrm{~mm} \mathrm{SL}$ ). Fig. 6. Nannobrachium regale (FAKU 200557, $183.2 \mathrm{~mm} \mathrm{SL}$ ). Fig. 7. Stenobrachius nannochir (FAKU 200385, $97.7 \mathrm{~mm} \mathrm{SL}$ ). Fig. 8. Laemonema longipes (FAKU 200791, 196.6 mm SL). Fig. 9. Eleginus gracilis (FAKU 200590, $206.4 \mathrm{~mm}$ SL). Fig. 10. Gadus chalcogrammus (FAKU 200562, $99.0 \mathrm{~mm} \mathrm{SL).} \mathrm{Fig.} 11$ Gadus macrocephalus (FAKU 200549, $262.5 \mathrm{~mm} \mathrm{SL})$. 


\section{TOHKAIRIN, A. ET AL.}
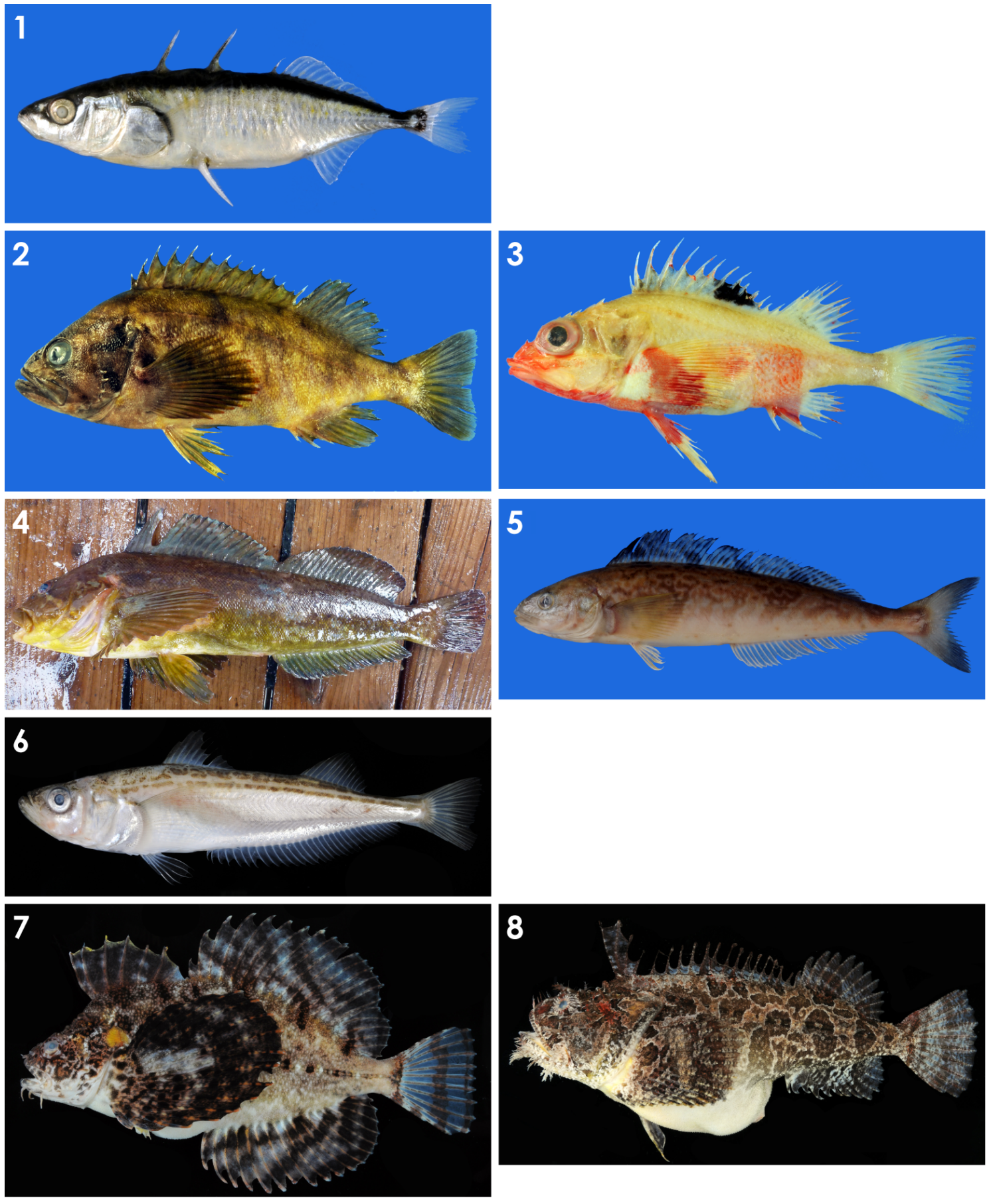

Plate III. Families Gasterosteidae (Fig. 1), Sebastidae (Fig. 2), Sebastolobidae (Fig. 3), Hexagrammidae (Figs. 4, 5), Trichodontidae (Fig. 6) and Hemitripteridae (Figs. 7, 8). Fig. 1. Gasterosteus nipponicus (FAKU 200688, $62.9 \mathrm{~mm} \mathrm{SL}$ ). Fig. 2. Sebastes glaucus (FAKU 200731, $191.4 \mathrm{~mm} \mathrm{SL}$ ). Fig. 3. Sebastolobus macrochir (FAKU 200753, $162.3 \mathrm{~mm} \mathrm{SL}$ ). Fig. 4. Hexagrammos lagocephalus. Fig. 5. Pleurogrammus azonus (FAKU 200797, $210.2 \mathrm{~mm}$ SL). Fig. 6. Arctoscopus japonicus (FAKU 201377, $178.9 \mathrm{~mm}$ SL). Fig. 7. Blepsias bilobus (FAKU201395, $171.4 \mathrm{~mm} \mathrm{SL}$ ). Fig. 8. Hemitripterus villosus (FAKU 201362, $165.8 \mathrm{~mm} \mathrm{SL}$ ). 

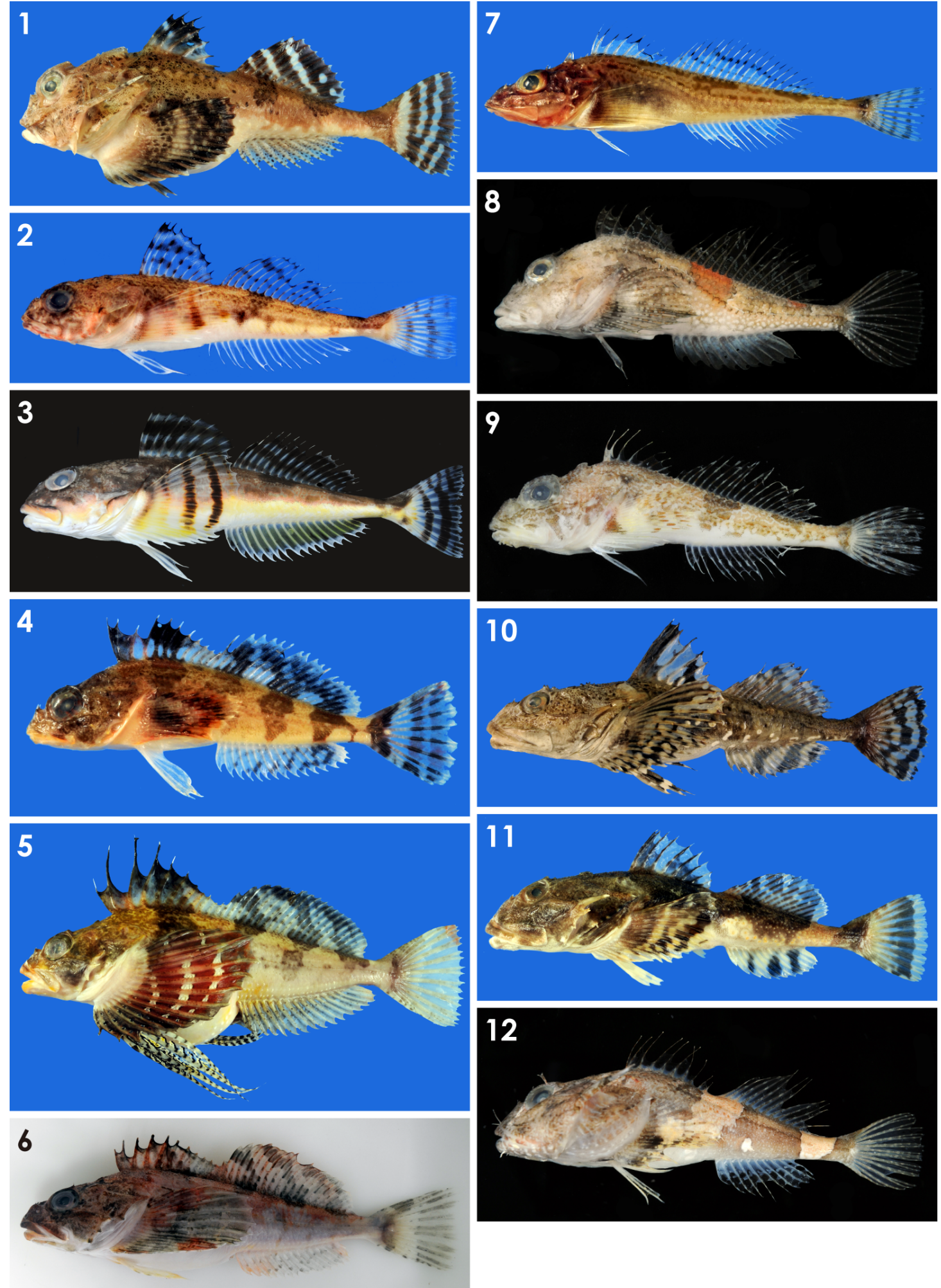

Plate IV. Family Cottidae (Figs. 1-12). Fig. 1. Enophrys diceraus (FAKU 200796, $189.0 \mathrm{~mm}$ SL). Fig. 2. Gymnocanthus detrisus (FAKU 200401, 85.9 mm SL). Fig. 3. Gymnocanthus herzensteini (FAKU 201358, $194.5 \mathrm{~mm}$ SL). Fig. 4. Hemilepidotus gilberti (FAKU 200800, $89.1 \mathrm{~mm}$ SL). Fig. 5. Hemilepidotus papilio, male (FAKU 200754, 246.9 mm SL). Fig. 6. Hemilepidotus papilio, female (FAKU 200455, $218.6 \mathrm{~mm} \mathrm{SL}$ ). Fig. 7. Icelus cataphractus (FAKU 200662, $177.7 \mathrm{~mm} \mathrm{SL}$ ). Fig. 8. Icelus gilberti (FAKU 201380, 83.7 mm SL). Fig. 9. Icelus ochotensis (FAKU 201397, $82.3 \mathrm{~mm}$ SL). Fig. 10. Myoxocephalus jaok (FAKU 200756, $278.7 \mathrm{~mm} \mathrm{SL}$ ). Fig. 11. Myoxocephalus polyacanthocephalus (FAKU 200755, $233.6 \mathrm{~mm} \mathrm{SL}$ ). Fig. 12. Taurocottus bergii (FAKU 201422, $107.6 \mathrm{~mm} \mathrm{SL}$ ). 


\section{TOHKAIRIN, A. ET AL.}
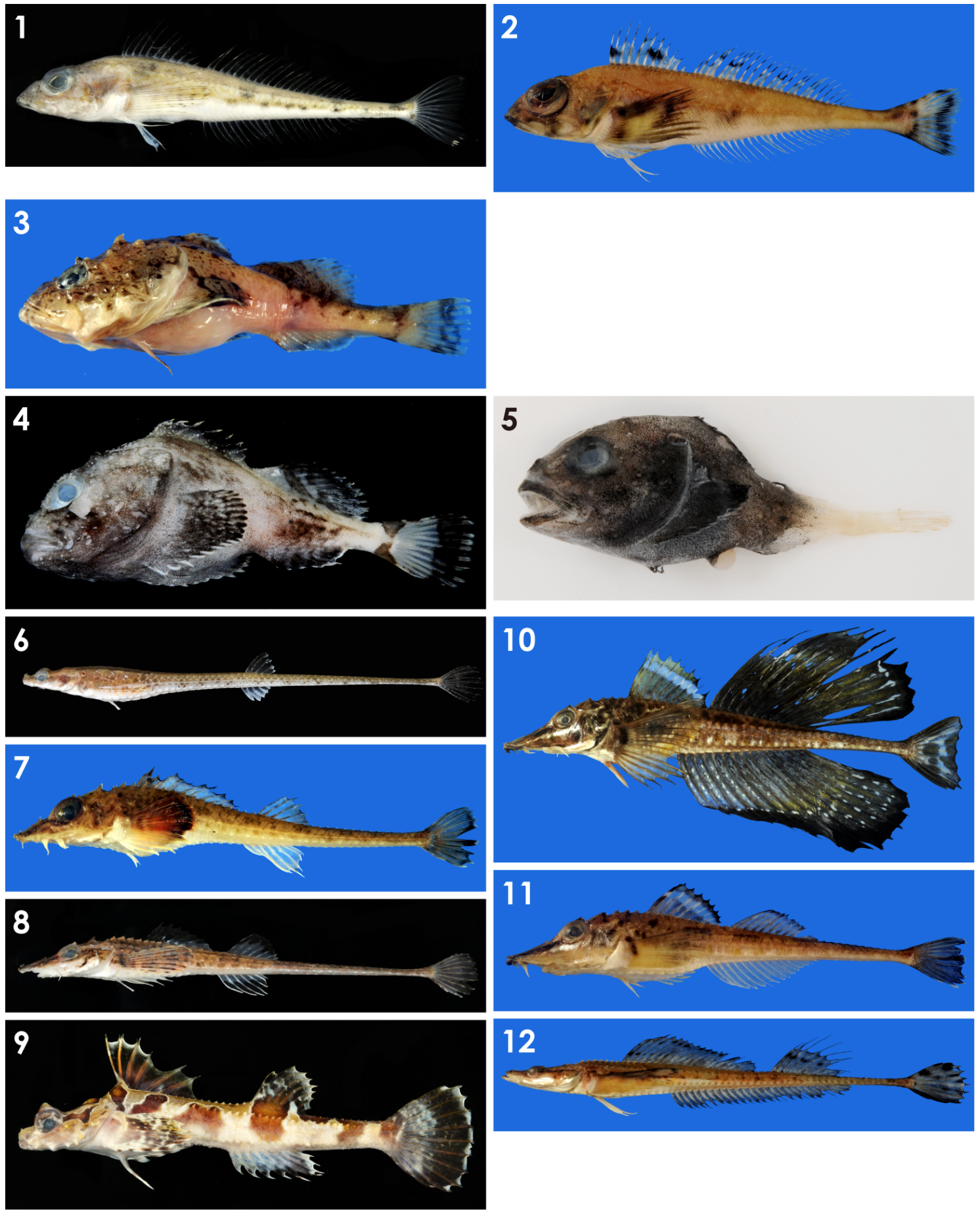

Plate V. Families Cottidae (Figs. 1, 2), Psychrolutidae (Figs. 3-5) and Agonidae (Figs. 6-12). Fig. 1. Triglops pingelii (FAKU 201383, $148.8 \mathrm{~mm} \mathrm{SL}$ ). Fig. 2. Triglops scepticus (FAKU200738, $147.8 \mathrm{~mm} \mathrm{SL}$ ). Fig. 3. Dasycottus setiger (FAKU 200664, $99.7 \mathrm{~mm} \mathrm{SL}$ ). Fig. 4. Malacocottus zonurus (FAKU 201382, $73.2 \mathrm{~mm} \mathrm{SL}$ ). Fig. 5. Malacocottus zonurus, small specimen with soft blackish body (FAKU 201398, 39.0 mm SL). Fig. 6. Aspidophoroides monopterygius (FAKU 201360, $153.2 \mathrm{~mm} \mathrm{SL}$ ). Fig. 7. Freemanichthys thompsoni (FAKU 200701, 153.0 mm SL). Fig. 8. Leptagonus leptorhynchus (FAKU 201412, $150.7 \mathrm{~mm}$ SL). Fig. 9. Percis japonica (FAKU 201433, $130.0 \mathrm{~mm} \mathrm{SL}$ ). Fig. 10. Podothecus sachi, male (FAKU 200686, $324.0 \mathrm{~mm}$ SL). Fig. 11. Podothecus sachi, female (FAKU 200580, $162.3 \mathrm{~mm}$ SL). Fig. 12. Tilesina gibbosa (FAKU 200669, $262.7 \mathrm{~mm} \mathrm{SL}$ ). 


\section{FISH OF KITAMI-YAMATO BANK, SOUTHERN SEA OF OKHOTSK}
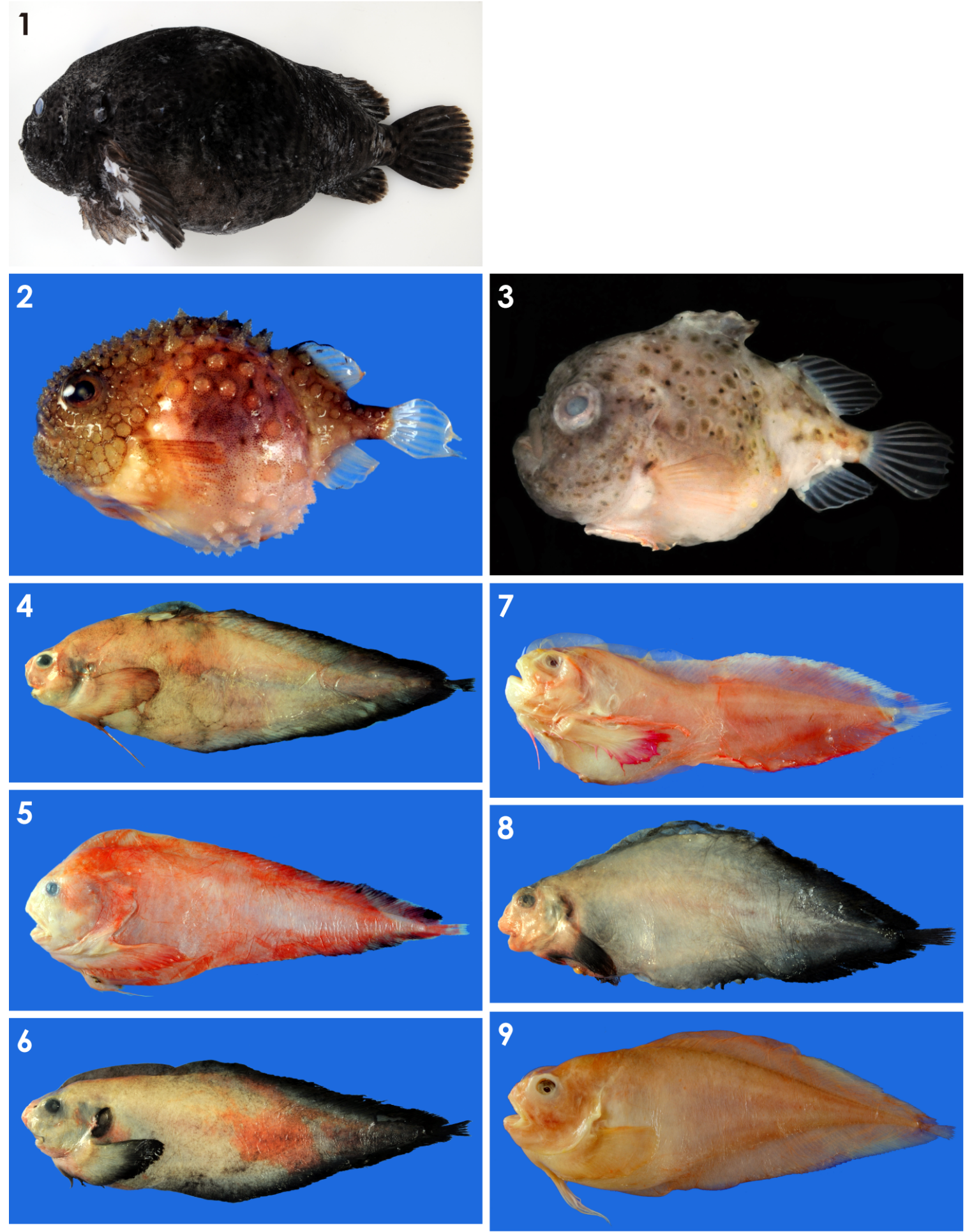

Plate VI. Families Cyclopteridae (Figs. 1-3) and Liparidae (Figs. 4-9). Fig. 1. Aptocyclus ventricosus (FAKU 201402, 209.4 mm SL). Fig. 2. Eumicrotremus asperrimus (FAKU 200679, 52.3 mm SL). Fig. 3. Eumicrotremus asperrimus, specimen with few bony tubercles and relatively distinct spinous dorsal fin (FAKU 201388, 49.9 mm SL). Fig. 4. Careproctus colletti (FAKU 200743, $250.1 \mathrm{~mm}$ SL). Fig. 5. Careproctus cyclocephalus (FAKU 200525, $284.4 \mathrm{~mm} \mathrm{SL}$ ). Fig. 6. Careproctus furcellus (FAKU 200744, $276.2 \mathrm{~mm}$ SL). Fig. 7. Careproctus macrodiscus (FAKU 200527, $207.2 \mathrm{~mm} \mathrm{SL}$ ). Fig. 8. Careproctus marginatus (FAKU 200477, $204.0 \mathrm{~mm} \mathrm{SL}$ ). Fig. 9. Careproctus rastrinus species complex (FAKU 200748, $252.5 \mathrm{~mm} \mathrm{SL})$. 

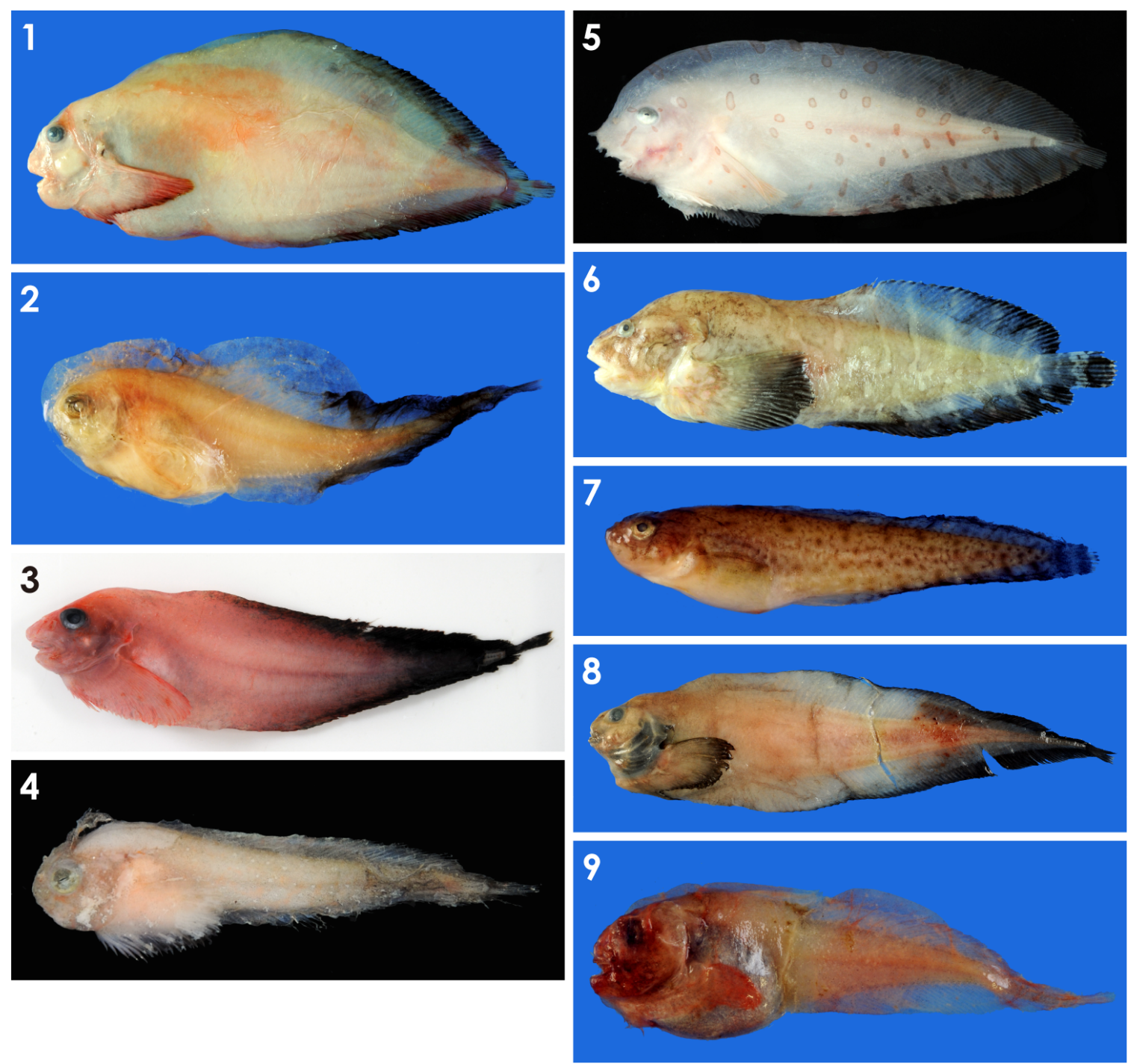

Plate VII. Family Liparidae (Figs. 1-9). Fig. 1. Careproctus roseofuscus (FAKU 200554, 276.9 mm SL). Fig. 2. Careproctus segaliensis (FAKU 200556, $75.0 \mathrm{~mm} \mathrm{SL}$ ). Fig. 3. Careproctus simus (FAKU 201401, $165.2 \mathrm{~mm} \mathrm{SL}$ ). Fig. 4. Careproctus sp. (FAKU 201379, $41.0 \mathrm{~mm}$ SL). Fig. 5. Crystallichthys matsushimae (FAKU 201410, 131.7 mm SL). Fig. 6. Liparis ochotensis (FAKU 200654, $215.7 \mathrm{~mm} \mathrm{SL).}$ Fig. 7. Liparis tessellatus (FAKU 200559, 82.9 mm SL). Fig. 8. Paraliparis grandis (FAKU 200481, 309.1 mm SL). Fig. 9. Paraliparis sp. (FAKU 200474, $85.7 \mathrm{~mm} \mathrm{SL}$ ). 


\section{FISH OF KITAMI-YAMATO BANK, SOUTHERN SEA OF OKHOTSK}
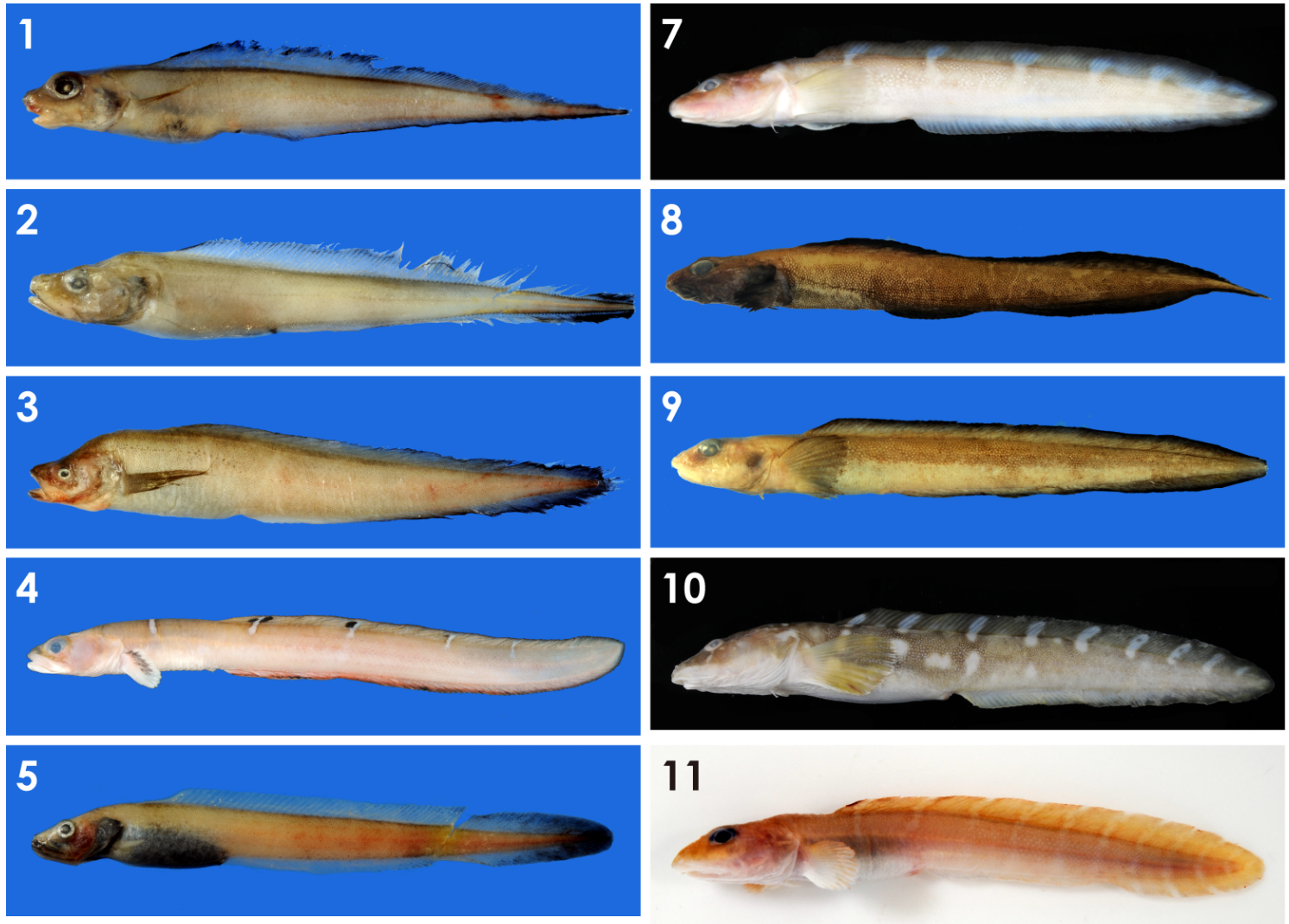

11
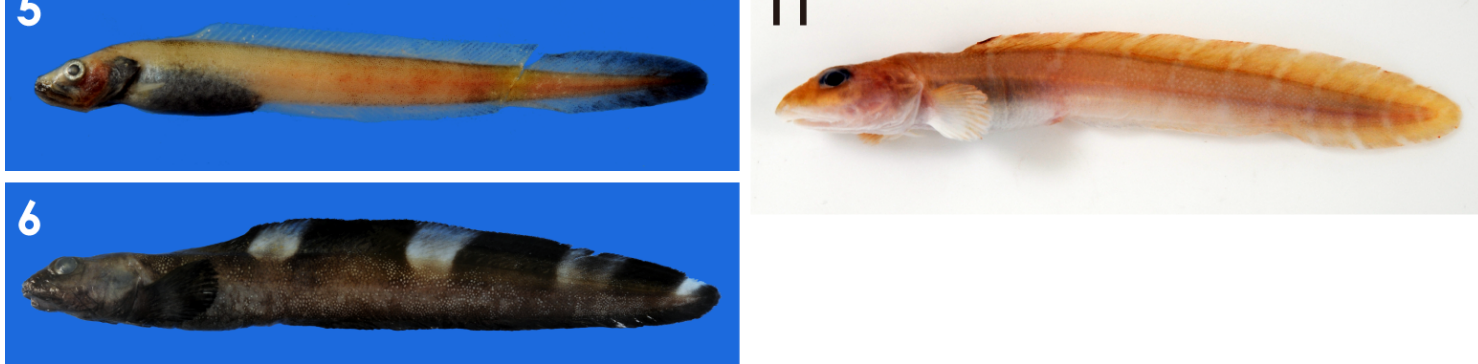

Plate VIII. Family Zoarcidae (Figs. 1-11). Fig. 1. Bothrocara hollandi (FAKU 200465, 189.2 mm SL). Fig. 2. Bothrocara zestum (FAKU 200785, caudal part damaged). Fig. 3. Bothrocarina microcephala (FAKU 200462, 238.1 mm SL). Fig. 4. Gymnelopsis ochotensis (FAKU 201443, 179.4 mm SL). Fig. 5. Lycodapus microchir (FAKU 200788, 122.6mm SL). Fig. 6. Lycodes albonotatus (FAKU 200466, 192.7 mm SL). Fig. 7. Lycodes matsubarai (FAKU 201367, $180.7 \mathrm{~mm} \mathrm{SL}$ ). Fig. 8. Lycodes microporus (FAKU 200372, $157.8 \mathrm{~mm} \mathrm{SL}$ ). Fig. 9. Lycodes pectoralis (FAKU 200567, $272.2 \mathrm{~mm} \mathrm{SL}$ ). Fig. 10. Lycodes tanakae (FAKU 201369, 262.1 mm SL). Fig. 11. Petroschmidtia teraoi (FAKU 201446, 102.5 mm SL). 

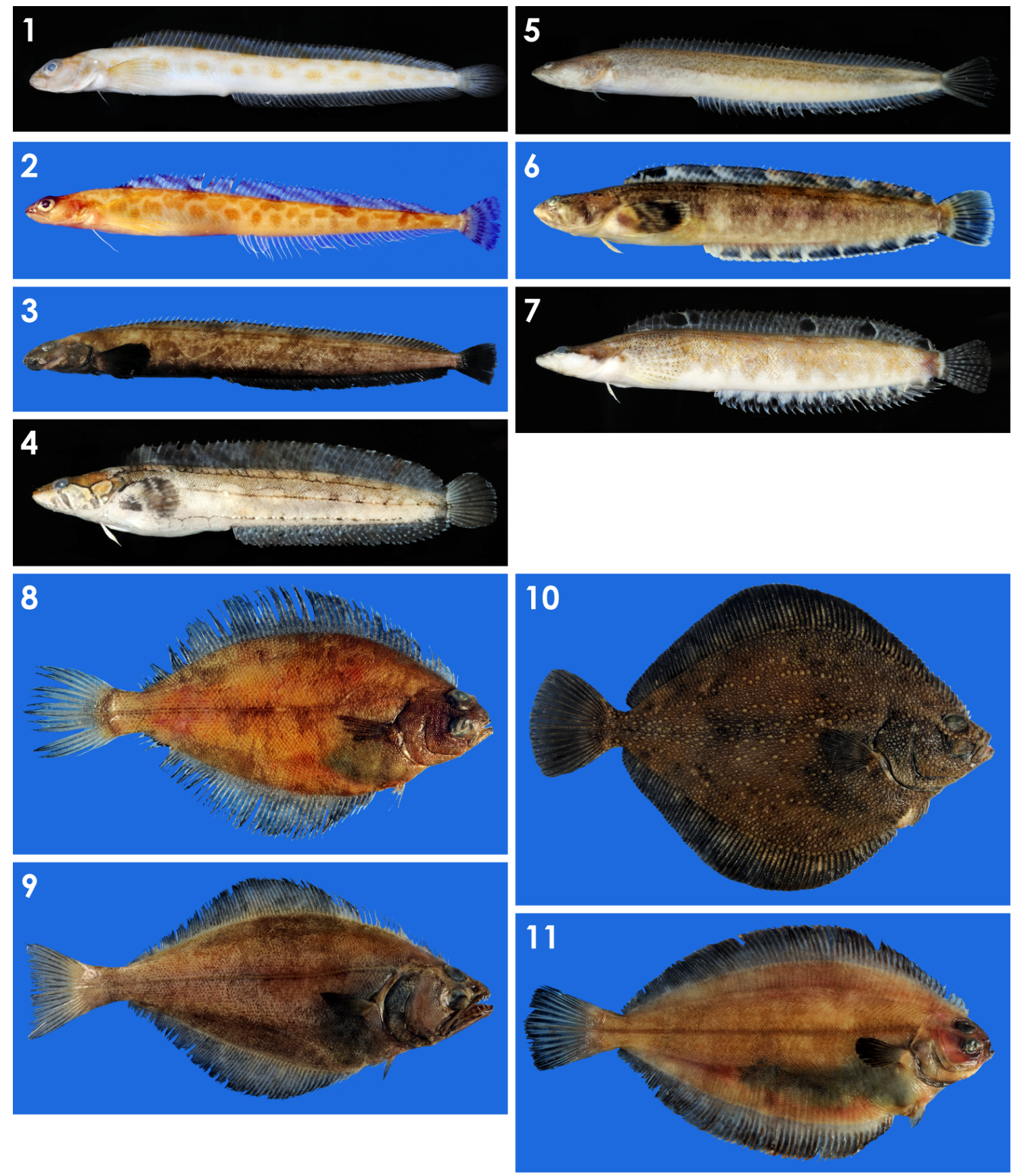

Plate IX. Families Stichaeidae (Figs. 1-7) and Pleuronectidae (Figs. 8-11). Fig. 1. Anisarchus medius (FAKU 201371, 159.8 mm SL). Fig. 2. Leptoclinus maculatus (FAKU 200801, 162.2 mm SL). Fig. 3. Lumpenella longirostris (FAKU 200468, $279.8 \mathrm{~mm} \mathrm{SL}$ ). Fig. 4. Stichaeopsis epallax (FAKU 201390, 144.3 mm SL). Fig. 5. Stichaeus grigorjewi (FAKU 201378, 109.7 mm SL). Fig. 6. Stichaeus nozawae (FAKU 200665, $224.3 \mathrm{~mm}$ SL). Fig. 7. Stichaeus ochriamkini (FAKU 201356, $130.6 \mathrm{~mm}$ SL). Fig. 8. Acanthopsetta nadeshnyi (FAKU 200723, $202.3 \mathrm{~mm}$ SL). Fig. 9. Atheresthes evermanni (FAKU 200558, $330.0 \mathrm{~mm} \mathrm{SL}$ ). Fig. 10. Clidoderma asperrimum (FAKU 200761, 261.3 mm SL). Fig. 11. Glyptocephalus stelleri (FAKU 200767, $245.3 \mathrm{~mm} \mathrm{SL}$ ). 

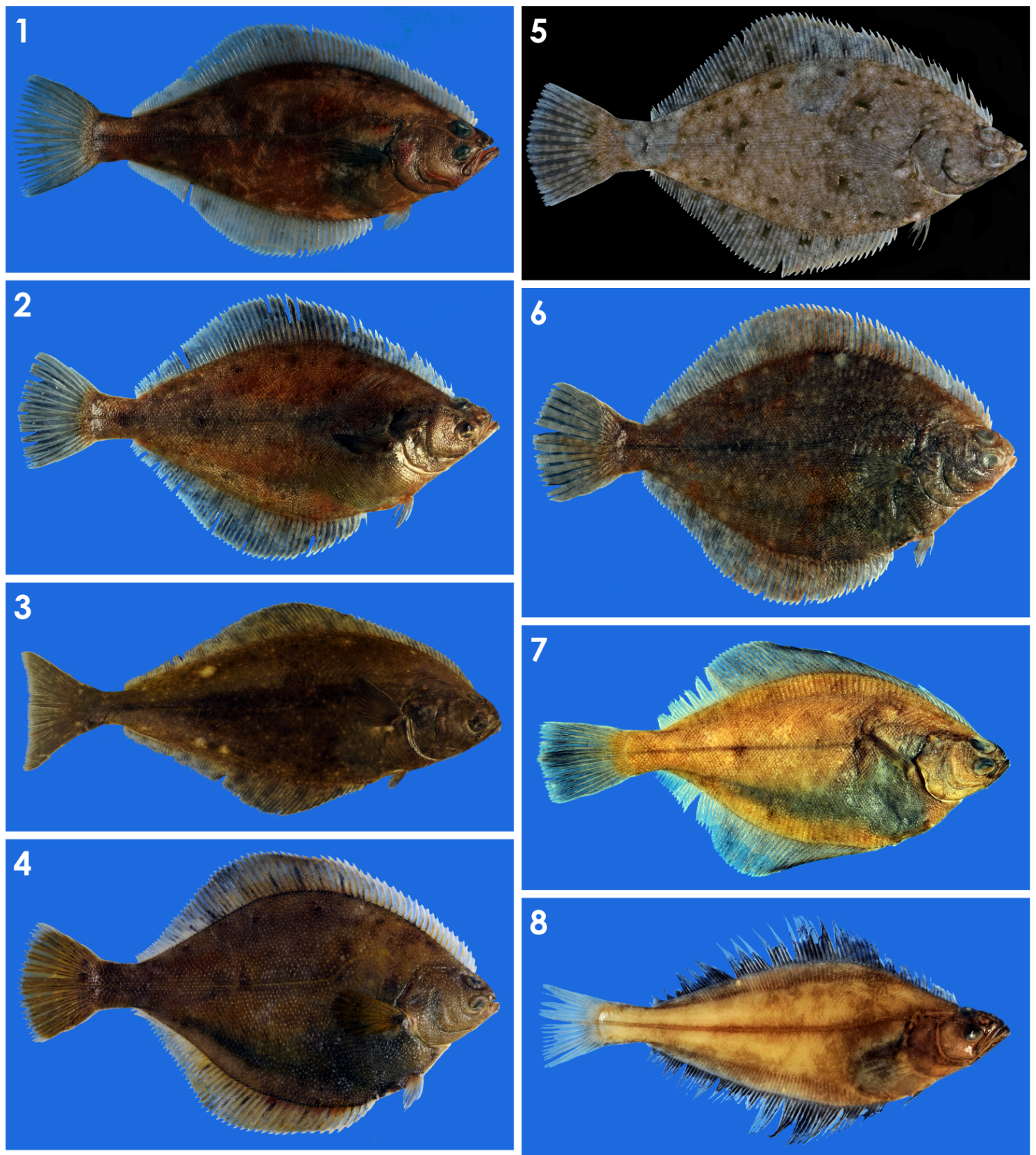

Plate X. Family Pleuronectidae (Figs. 1-8). Fig. 1. Hippoglossoides dubius (FAKU 200720, $210.3 \mathrm{~mm}$ SL). Fig. 2. Hippoglossoides pinetorum (FAKU 200721, $226.6 \mathrm{~mm} \mathrm{SL}$ ). Fig. 3. Hippoglossus stenolepis (FAKU 200461, $389.0 \mathrm{~mm}$ SL). Fig. 4. Pleuronectes asper (FAKU 200773, $267.7 \mathrm{~mm}$ SL). Fig. 5. Pleuronectes herzensteini (FAKU 201361, $186.3 \mathrm{~mm}$ SL). Fig. 6. Pleuronectes mochigarei (FAKU 200762, $252.8 \mathrm{~mm} \mathrm{SL}$ ). Fig. 7. Pleuronectes sakhalinensis (FAKU 200782, $171.3 \mathrm{~mm} \mathrm{SL}$ ). Fig. 8. Reinhardtius hippoglossoides (FAKU 200729, $123.4 \mathrm{~mm} \mathrm{SL}$ ). 